\title{
Testing supersymmetry at the LHC through gluon-fusion production of a slepton pair
}

\author{
F. Borzumati ${ }^{1}$ and K. Hagiwara ${ }^{2}$ \\ ${ }^{1}$ Department of Physics, National Taiwan University, Taipei 10617, Taiwan \\ ${ }^{2}$ KEK Theory Division, and Sokendai, Tsukuba 305-0801, Japan
}

\begin{abstract}
Renormalizable quartic couplings among new particles are typical of supersymmetric models. Their detection could provide a test for supersymmetry, discriminating it from other extensions of the Standard Model. Quartic couplings among squarks and sleptons, together with the $\mathrm{SU}(3)$ gauge couplings for squarks, allow a new realization of the gluon-fusion mechanism for pair-production of sleptons at the one-loop level. The corresponding production cross section, however, is at most of $\mathcal{O}(1) \mathrm{fb}$ for slepton and squark masses of $\mathcal{O}(100) \mathrm{GeV}$. We then extend our investigation to the gluon-fusion production of sleptons through the exchange of Higgs bosons. The cross section is even smaller, of $\mathcal{O}(0.1) \mathrm{fb}$, if the exchanged Higgs boson is considerably below the slepton-pair threshold, but it is enhanced when it is resonant. It can reach $\mathcal{O}(10)$ fb for the production of sleptons of same-chirality, exceeding these values for $\widetilde{\tau}$ 's of opposite-chirality, even when chirality-mixing terms in the squark sector are vanishing. The cross section can be further enhanced if these mixing terms are nonnegligible, providing a potentially interesting probe of the Higgs sector, in particular of parameters such as $A, \mu$, and $\tan \beta$.
\end{abstract}




\section{Introduction}

It has been realized since quite some time that several extensions of the Standard Model (SM) share similar signatures at the LHC, as, for example, that of jets, leptons, and missing transverse energy [1. This is because such extensions typically have partners of the SM, with gauge quantum numbers equal to those of the SM particles [2]. Supersymmetric models, extra-dimensional ones, and little Higgs models are indeed of this type. Among them, supersymmetric models stand out because the partners have spins that differ by 1/2 from those of the SM particles, whereas in the other extensions have the same spins. This has prompted several proposal of methods for determining the spin of heavy unstable particles [3].

Thus, supersymmetric models are also the only ones, at least so far, in which there exist irreducible renormalizable interactions of four matter partners, of type $\phi^{4}$. Such interactions may, therefore, offer a new handle to discriminate Supersymmetry (SUSY) from other extensions of the SM.

The LHC being a hadronic machine, quartic couplings of sleptons, $\left(\widetilde{l}^{*} \widetilde{l}\right)^{2}$, contribute only to processes whose cross sections are suppressed by many powers of the electroweak couplings. The signals of quartic couplings of squarks, $\left(\widetilde{q}^{*} \widetilde{q}\right)^{2}$, may not be easily disentangled from those due to a pair of squarks. The first, simplest scrutiny of quartic couplings, therefore, may be that of couplings of two sleptons and two squarks $\left(\widetilde{q}^{*} \widetilde{q}\right)(\widetilde{l} * \widetilde{l})$. Combined with the strong interaction vertices of squarks and gluons, these give rise, at the quantum level, to the effective couplings $g g \widetilde{l} * \widetilde{l}$, and therefore to the gluon-initiated production of a pair of slepton.

Since Higgs bosons couple to both, a pair of fermions and a pair of scalars, reducible couplings of four SM partners, two quark partners and two lepton partners, can be built through the exchange of Higgs bosons also in models in which the partner spins do not differ from those of the SM particles. Thus, the gluon-fusion production of a pair of lepton partners is not a special feature of supersymmetric models. The possibility of discriminating SUSY through this type of studies strongly relies on the impact that the irreducible quartic couplings of supersymmetric models can have.

To the best of our knowledge, the one-loop process $g g \widetilde{l}^{*} \widetilde{l}$ via quartic couplings, was never studied before. Surprisingly, very little exists in the literature also about the same process induced by the exchange of a Higgs boson, $g g \rightarrow H^{0} \rightarrow \widetilde{l}{ }^{*}$. It was studied in Ref. 4] for $H^{0}=h$, the lightest Higgs bosons. Since this has a mass clearly below the slepton-pair threshold, the cross section was found to be very small. The study of this process was put aside until the authors of Ref. [5] realized that the cross section could increase if $H^{0}$ is one of the two heavier neutral Higgs bosons $H^{0}=H, A$. Neverheless, not much attention has been given to this proposal.

Although suppressed by a loop factor and by the coupling $\alpha_{s}$ with respect to that of the standard Drell-Yan production $q \bar{q} \rightarrow \widetilde{l}^{*} \widetilde{l}$, the gluon-fusion cross sections may be favoured by the fact that, at the LHC, gluon-initiated processes may dominate over the quark-initiated ones. Given the importance of the quartic couplings that may be probed, 
it is worth studying them in detail.

The paper is organized as follows. In Sec. 2 we study the slepton pair production processes from gluon fusion induced by quartic couplings. In Sec. 3 we give the complete one-loop level results, including also the contribution from the Higgs boson-exchange. We summarize our findings in Sec. 4 .

\section{Gluon-fusion production from quartic couplings}

\subsection{Cross section}

The relevant quartic interactions in the scalar potential of the Minimal Supersymmetric Standard Model (MSSM) consist of D-term interactions, proportional to gauge couplings squared, and F-terms interactions, proportional to Yukawa couplings squared. These are respectively collected in:

$$
V_{D} \supset d^{\widetilde{l}_{i} \widetilde{l}_{i}, \widetilde{q}_{n} \widetilde{q}_{n}}\left(\widetilde{q}_{n}^{*} \widetilde{q}_{n}\right)\left(\widetilde{l}_{i}^{*} \widetilde{l}_{i}\right), \quad V_{F} \supset f^{\widetilde{l}_{i} \widetilde{l}_{j}, \widetilde{q}_{m} \widetilde{q}_{n}}\left(\widetilde{q}_{m}^{*} \widetilde{q}_{n}\right)\left(\widetilde{l}_{i}^{*} \widetilde{l}_{j}\right)+\text { H.c., }
$$

where $\widetilde{q}_{n}$ is any of the fields $\widetilde{u}_{L}, \widetilde{d}_{L}, \widetilde{u}_{R}, \widetilde{d}_{R}$, of any generation, and $\widetilde{l}_{i}$ is either the charged component of the $\mathrm{SU}(2)_{L}$ doublet $\left(\widetilde{\nu}_{L}, \widetilde{l}_{L}\right)$ with hypercharge $-1 / 2$, or the $\mathrm{SU}(2)_{L}$ singlet $\widetilde{l}_{R}$ with hypercharge -1 , also of any generation. The coefficients $d^{\widetilde{l}_{i} \widetilde{l}_{i}, \widetilde{q}_{n} \widetilde{q}_{n}}$ and $f^{\widetilde{l}_{i} \widetilde{l}_{j}, \widetilde{q}_{m} \widetilde{q}_{n}}$ are listed in Table 1, where $g_{1}$ and $g_{2}$ are the coupling constants of $\mathrm{U}(1)_{Y}$ and $\mathrm{SU}(2)_{L}$, with the normalization $g_{1} \cos \theta_{W}=g_{2} \sin \theta_{W}=e=\sqrt{4 \pi \alpha}$. The Yukawa coupling matrices, denoted by $h_{U}, h_{D}$, and $h_{E}$, are assumed to be diagonal and real, with only the $\{3,3\}$ element large enough to be relevant for this discussion.

These quartic couplings, together with the SU(3) interactions for squarks:

$$
K_{q_{n}^{*} q_{n} g(g)}=-g_{S} \widetilde{q}_{n}^{*}\left(\frac{\lambda^{a}}{2}\right) \stackrel{\leftrightarrow}{\partial^{\mu}} \widetilde{q}_{n} g_{\mu}^{a}+g_{S}^{2} \widetilde{q}_{n}^{*}\left(\frac{\lambda^{a}}{2} \frac{\lambda^{b}}{2}\right) \widetilde{q}_{n} g_{\mu}^{a} g^{b \mu}
$$

allow a realization of the effective couplings $g g \widetilde{l}_{i}^{*} \widetilde{l}_{j}$ at the one-loop level, different from those due to the mediation of an s-channel Higgs boson. In this expression, $g_{S}$ is the strong coupling and $(1 / 2)\left\{\lambda^{a}\right\}$ are the generators of $\mathrm{SU}(3)$.

Examples of loop diagrams giving rise to the effective interactions $g g \widetilde{l}_{i}^{*} \widetilde{l}_{j}$ through quartic couplings are given in Fig. 1. The corresponding parton-level cross section is

$$
\widehat{\sigma}_{\mathrm{QC}}\left(g g \rightarrow \widetilde{l}_{i}^{*} \widetilde{l}_{j}\right)=\frac{\alpha_{s}^{2}}{(16 \pi)^{3}} \frac{\lambda^{1 / 2}\left(\hat{s}, \widetilde{m}_{l_{i}}, \widetilde{m}_{l_{j}}\right)}{\hat{s}}\left|\widetilde{A}_{\mathrm{QC}}^{\widetilde{l}_{i} \tilde{l}_{j}}\right|^{2}
$$

where $\lambda^{1 / 2}\left(\hat{s}, m_{1}, m_{2}\right)=\left[1-\left(m_{1}+m_{2}\right)^{2} / \hat{s}\right]^{1 / 2}\left[1-\left(m_{1}-m_{2}\right)^{2} / \hat{s}\right]^{1 / 2}$, for the production of two particles with mass $m_{1}$ and $m_{2}$, or simply $\lambda^{1 / 2}(\hat{s}, m)=\left[1-4 m^{2} / \hat{s}\right]^{1 / 2}$, the usual $\beta$ factor, if $m_{1}=m_{2}=m$. A dependence on the produced slepton masses is also hidden in $\hat{s}$, as 


\begin{tabular}{cccc}
\hline \hline$l_{i}^{*} l_{j}$ & $q_{m}^{*} q_{n}$ & $d^{\widetilde{l}_{i} \widetilde{l}_{i}, \widetilde{q}_{n} \widetilde{q}_{n}}$ & $f^{\widetilde{l}_{i} \widetilde{\tau}_{j}, \widetilde{q}_{m} \widetilde{q}_{n}}$ \\
\hline$\widetilde{l}_{L}^{*} \widetilde{l}_{L}$ & $\widetilde{u}_{L}^{*} \widetilde{u}_{L}$ & $-\frac{g_{2}^{2}}{4}-\frac{g_{1}^{2}}{12}$ & - \\
$\widetilde{l}_{L}^{*} \widetilde{l}_{L}$ & $\widetilde{d}_{L}^{*} \widetilde{d}_{L}$ & $+\frac{g_{2}^{2}}{4}-\frac{g_{1}^{2}}{12}$ & - \\
$\widetilde{l}_{L}^{*} \widetilde{l}_{L}$ & $\widetilde{d}_{R}^{*} \widetilde{d}_{R}$ & $-\frac{g_{1}^{2}}{6}$ & - \\
$\widetilde{l}_{L}^{*} \widetilde{l}_{L}$ & $\widetilde{u}_{R}^{*} \widetilde{u}_{R}$ & $+\frac{g_{1}^{2}}{3}$ & - \\
\hline$\widetilde{l}_{R}^{*} \widetilde{l}_{R}$ & $\widetilde{u}_{L}^{*} \widetilde{u}_{L}$ & $+\frac{g_{1}^{2}}{6}$ & - \\
$\widetilde{l}_{R}^{*} \widetilde{l}_{R}$ & $\widetilde{d}_{L}^{*} \widetilde{d}_{L}$ & $+\frac{g_{1}^{2}}{6}$ & - \\
$\widetilde{l}_{R}^{*} \widetilde{l}_{R}$ & $\widetilde{d}_{R}^{*} \widetilde{d}_{R}$ & $+\frac{g_{1}^{2}}{3}$ & - \\
$\widetilde{l}_{R}^{*} \widetilde{l}_{R}$ & $\widetilde{u}_{R}^{*} \widetilde{u}_{R}$ & $-\frac{2}{3} g_{1}^{2}$ & - \\
\hline$\widetilde{\tau}_{L}^{*} \widetilde{\tau}_{R}$ & $\widetilde{b}_{R}^{*} \widetilde{b}_{L}$ & - & $\left(h_{D}\right)_{3,3}\left(h_{E}\right)_{3,3}$ \\
\hline \hline
\end{tabular}

Table 1: Coefficients $d^{\widetilde{l}_{i} \widetilde{l}_{i}, \widetilde{q}_{n} \widetilde{q}_{n}}$ and $f^{\widetilde{l}_{i} \widetilde{l}_{j}, \widetilde{q}_{m} \widetilde{q}_{n}}$ for quartic squark-charged slepton interactions, from $D$ - and F-terms. The conjugated term $\left(\widetilde{\tau}_{R}^{*} \widetilde{\tau}_{L}\right)\left(\widetilde{b}_{L}^{*} \widetilde{b}_{R}\right)$ also exists, but given our approximation for the Yukawa couplings, it has an F-term coefficient equal to that of $\left(\widetilde{\tau}_{L}^{*} \widetilde{\tau}_{R}\right)\left(\widetilde{b}_{R}^{*} \widetilde{b}_{L}\right)$.

the minimum value that $\hat{s}$ can acquire is $\hat{s}_{\min }=\left(\widetilde{m}_{l_{i}}+\widetilde{m}_{l_{j}}\right)^{2}$. The truncated amplitude $\widetilde{A}_{\mathrm{QC}}^{\tilde{i}_{i} \tilde{l}_{j}}$ is calculated hereafter.

Since the strong interactions do not change chirality, contributions to $\widetilde{A}_{\mathrm{QC}}^{\widetilde{l}_{i} \widetilde{J}_{j}}$ from Fterms are possible only for nonvanishing chirality-mixing terms in the $\widetilde{b}$ squark sector. These are included in the mass-mixing terms of the scalar potential:

$$
V_{\text {ch-mix }} \supset \widetilde{u}_{R}^{*} \widetilde{m}_{U, \mathrm{RL}}^{2} \widetilde{u}_{L}+\widetilde{d}_{R}^{*} \widetilde{m}_{D, \mathrm{RL}}^{2} \widetilde{d}_{L}+\widetilde{l}_{R}^{*} \widetilde{m}_{E, \mathrm{RL}}^{2} \widetilde{l}_{L}+\text { H.c., }
$$

which contribute, together with those in the chirality-conserving soft mass terms:

$$
V_{2}^{\mathrm{soft}}=\left(\widetilde{u}_{L}^{*}, \widetilde{d}_{L}^{*}\right) \widetilde{m}_{Q}^{2}\left(\begin{array}{c}
\widetilde{u}_{L} \\
\widetilde{d}_{L}
\end{array}\right)+\widetilde{u}_{R}^{*} \widetilde{m}_{U}^{2} \widetilde{u}_{R}+\widetilde{d}_{R}^{*} \widetilde{m}_{D}^{2} \widetilde{d}_{R}+\left(\widetilde{\nu}_{L}^{*}, \widetilde{l}_{L}^{*}\right) \widetilde{m}_{L}^{2}\left(\widetilde{\nu}_{L}\right)+\widetilde{l}_{L}^{*} \widetilde{m}_{E}^{2} \widetilde{l}_{R},
$$

to the up- and down-squark as well as slepton mass matrices. In our discussion, the parameters $\widetilde{m}_{Q}^{2}, \widetilde{m}_{U}^{2}, \widetilde{m}_{D}^{2}, \widetilde{m}_{L}^{2}, \widetilde{m}_{E}^{2}$ are $3 \times 3$ diagonal Hermitian matrices and the chiralitymixing parameters $\widetilde{m}_{U, \mathrm{RL}}^{2}, \widetilde{m}_{D, \mathrm{RL}}^{2}, \widetilde{m}_{E, \mathrm{RL}}^{2}$ are

$$
\widetilde{m}_{U, \mathrm{RL}}^{2}=\frac{\left(v_{u} A_{U}-\mu v_{d}\right) h_{U}}{\sqrt{2}}, \quad \widetilde{m}_{D, \mathrm{RL}}^{2}=\frac{\left(v_{d} A_{D}-\mu v_{u}\right) h_{D}}{\sqrt{2}}, \quad \widetilde{m}_{E, \mathrm{RL}}^{2}=\frac{\left(v_{d} A_{E}-\mu v_{u}\right) h_{E}}{\sqrt{2}},
$$



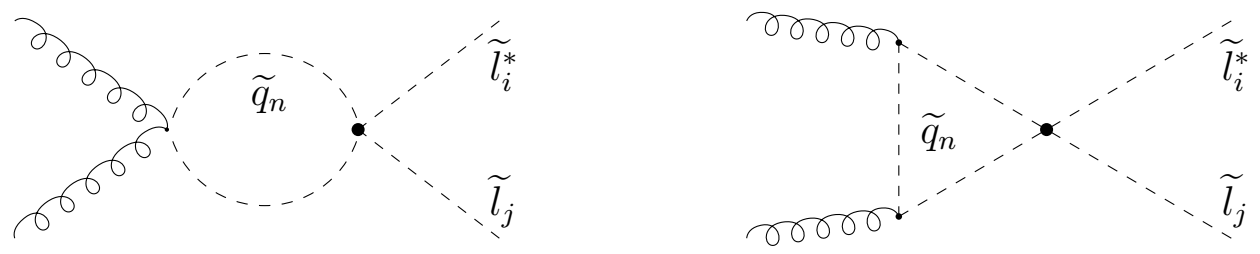

Figure 1: Diagrams contributing to the effective couplings gg $\widetilde{l}_{i}^{*} \widetilde{l}_{j}$. The big dot indicates a squark-slepton quartic coupling.

where $v_{d}, v_{u}$ are the vacuum expectation values of the neutral component in the two Higgs doublets $H_{d}$ and $H_{u}$, with $\tan \beta=v_{u} / v_{d}$ and $v^{2}=v_{d}^{2}+v_{u}^{2}$. (Our normalization is such that $M_{W}=g v / 2$.) The parameters $A_{U}, A_{D}$, and $A_{E}$ are the massive couplings of the trilinear soft terms holomorphic in the Higgs fields. We neglect also here any possible flavor mixing, i.e. $A_{U}, A_{D}$, and $A_{E}$ are, for our purposes, diagonal matrices, with real elements, and the terms in $V_{\mathrm{ch}-\text { mix }}$ are assumed to be sizable only for third-generation squarks.

If all chirality-mixing terms in $V_{\mathrm{ch}-\text { mix }}$ are negligible with respect to the soft mass squared in $V_{2}^{\text {soft }}$, in which case current eigenstates coincide to a good approximation with mass eigenstates, only same-chirality sleptons can be produced, induced by D-terms only. The corresponding truncated amplitude $\widetilde{A}_{\mathrm{QC}}^{l_{i} l_{i}}$ has the simple form:

$$
\widetilde{A}_{\mathrm{QC}}^{\widetilde{l}_{i} \widetilde{l}_{i}}=-\sum_{q=u, d}\left[d^{\widetilde{l}_{i} \widetilde{l}_{i}, \widetilde{q}_{L} \widetilde{q}_{L}} S\left(\hat{s} ; \widetilde{m}_{q_{L}}^{2}\right)+d^{\widetilde{l}_{i} \widetilde{l}_{i}, \widetilde{q}_{R} \widetilde{q}_{R}} S\left(\hat{s} ; \widetilde{m}_{q_{R}}^{2}\right)\right]
$$

where $l_{i}$ labels the type of produced sleptons, $l_{i}=\left\{l_{L}, l_{R}\right\}$, with $l=e, \mu, \tau$, and the sum in $q$ is understood to be over all three generations: $u \rightarrow u, c, t$, and $d \rightarrow d, s, b$. The loop function $S\left(\hat{s} ; \widetilde{m}_{q_{n}}^{2}\right)$ depends on $\hat{s}$ and $\widetilde{m}_{q_{n}}^{2}$ only through their ratio:

$$
S\left(\hat{s} ; m^{2}\right)=-1+\tau f(\tau), \quad \tau \equiv 4 m^{2} / \hat{s},
$$

where $f(\tau)$ is defined as:

$$
f(\tau)=\left\{\begin{array}{ll}
-\frac{1}{4}\left[\log \left(\frac{1+\sqrt{1-\tau}}{1-\sqrt{1-\tau}}\right)-i \pi\right]^{2} & \tau<1 \\
+\arcsin ^{2}\left(\frac{1}{\sqrt{\tau}}\right) & \tau \geq 1
\end{array} .\right.
$$

As mentioned already, there is an implicit dependence on the mass of the produced sleptons, due to the fact that $\hat{s}_{\text {min }}$, the minimal value that $\hat{s}$ can have, depends on it.

A quick inspection of Table 1 shows that:

$$
\sum_{\widetilde{q}_{n}} d^{\widetilde{l}_{L} \widetilde{l}_{L}, \widetilde{q}_{n} \widetilde{q}_{n}}=\sum_{\widetilde{q}_{n}} d^{\widetilde{l}_{R} \widetilde{l}_{R}, \widetilde{q}_{n} \widetilde{q}_{n}}=0,
$$

for each generation of squarks. This is no surprise, as, for each generation, the sums of the isospin and the weak hypercharge of all quarks and leptons vanish in the SM. The 
consequence of these sum rules is that, in the limit of degenerate squark masses, $\widetilde{A}_{\mathrm{QC}}^{\widetilde{l}_{L} \widetilde{l}_{L}}$ and $\widetilde{A}_{\mathrm{QC}}^{\widetilde{l}_{R} \widetilde{l}_{R}}$ vanish identically.

A complete degeneracy of all squarks of the same generation, is however unlikely. Sizable differences between the soft mass of the right-handed and left-handed squarks at low energy are present in most supersymmetric models. To give an example, in models with universal boundary conditions for soft masses at some high scale, typically the Planck mass or the scale of grand unification, a sufficient splitting between the soft mass of the right- and left-handed squarks at the electroweak scale is induced by the fact that, contrary to $\widetilde{m}_{U}^{2}$ and $\widetilde{m}_{D}^{2}, \widetilde{m}_{Q}^{2}$ feels the effect of the $\mathrm{SU}(2)_{L}$ gaugino during the downward evolution. In typical gauge-mediation models, with a weekly coupled messenger sector, a splitting is already present at the scale of SUSY breaking and may be further enlarged by a subsequent evolution down to the electroweak scale. Thus, the value of $\widetilde{A}_{\mathrm{QC}}^{\widetilde{l}_{i} \widetilde{l}_{i}}$ can be easily lifted from zero.

If some of the terms in $V_{\mathrm{ch}-\text { mix }}$ cannot be neglected, the expression for $\widetilde{A}_{\mathrm{QC}}^{\widetilde{l}_{i} \widetilde{l}_{i}}$ in Eq. (7) is not valid. Indeed, even for small values of $\tan \beta$, the above approximation may fail for $\tilde{t}$ squark states, but also for other squarks states, for large values of $A_{U}$ or $A_{D}$. In this case, the cross section must be expressed in terms of the sfermion mass eigenstates: $\widetilde{f}_{1}=\widetilde{f}_{L} \cos \theta_{f}+\widetilde{f}_{R} \sin \theta_{f}, \widetilde{f}_{2}=\widetilde{f}_{L} \sin \theta_{f}-\widetilde{f}_{R} \cos \theta_{f}$, and the mixing angles $\theta_{f}$, whose sine and cosine are often abbreviated in the following as $s_{f}$ and $c_{f}$, respectively. That for $\tilde{t}$ squarks is defined by the relation:

$$
\cos 2 \theta_{t}=\frac{\left(\widetilde{m}_{\bar{U}}^{2}\right)_{3,3}-\left(\widetilde{m}_{Q}^{2}\right)_{3,3}+\left(\Delta_{D}\right)_{u}}{\widetilde{m}_{t_{2}}^{2}-\widetilde{m}_{t_{1}}^{2}},
$$

where the numerator is the difference between the often called right-right and left-left diagonal entries in the $\tilde{t}$ squark mass matrix. In particular, $\left(\Delta_{D}\right)_{u}$ is the difference of the D-term contributions to these entries. A similar formula holds for $\widetilde{b}$ squarks, with the replacements $\left(\widetilde{m}_{\bar{U}}^{2}\right)_{3,3} \rightarrow\left(\widetilde{m}_{\bar{D}}^{2}\right)_{3,3}, \widetilde{m}_{t_{i}}^{2} \rightarrow \widetilde{m}_{b_{i}}^{2}(i=1,2)$, and $\left(\Delta_{D}\right)_{u} \rightarrow\left(\Delta_{D}\right)_{d}$. It can be easily adapted also to the case of $\widetilde{\tau}$ sleptons. The explicit expressions for $\left(\Delta_{D}\right)_{u}$, $\left(\Delta_{D}\right)_{d}$, and $\left(\Delta_{D}\right)_{l}$ can be found in any pedagogical review of SUSY. (See for example Ref. 9].) For $\tan \beta \gtrsim 3$, which is always assumed in this paper, these quantities can be approximated as

$$
\left(\Delta_{D}\right)_{u}=+\frac{1}{6} M_{Z}^{2}, \quad\left(\Delta_{D}\right)_{d}=-\frac{1}{3} M_{Z}^{2}, \quad\left(\Delta_{D}\right)_{l}=0 .
$$

Notice that the usual two-fold ambiguity in the definition of $\cos 2 \theta_{t}$ has been implicitly removed with the assumption $\left(\widetilde{m}_{\bar{U}}^{2}\right)_{3,3}<\left(\widetilde{m}_{Q}^{2}\right)_{3,3}-(1 / 6) M_{Z}^{2}$. In the limit of vanishing chirality-mixing terms for a sfermion $f, \cos \theta_{f} \rightarrow 0, \widetilde{f}_{1} \rightarrow \widetilde{f}_{R}$, and $\widetilde{f}_{2} \rightarrow \widetilde{f}_{L}$.

The generalization of $\widetilde{A}_{\mathrm{QC}}^{\widetilde{l}_{i} \widetilde{l}_{i}}$ to the case of nonnegligible chirality-mixing terms in the squark sector is then straightforward:

$$
\begin{array}{r}
\widetilde{A}_{\mathrm{QC}}^{\widetilde{l}_{i} \widetilde{l}_{i}=-\sum_{q=u, d}[}\left[\left(d^{\tilde{l}_{i} \widetilde{l}_{i}, \widetilde{q}_{L} \widetilde{q}_{L}} c_{q}^{2}+d^{{\widetilde{l_{i}}}_{i} \widetilde{l}_{i}, \widetilde{q}_{R} \widetilde{q}_{R}} s_{q}^{2}\right) S\left(\hat{s} ; m_{\widetilde{q}_{1}}^{2}\right)+\right. \\
\left.\left(d^{\widetilde{l}_{i} \widetilde{l}_{i}, \widetilde{q}_{L} \widetilde{q}_{L}} s_{q}^{2}+d^{\widetilde{l}_{i} \widetilde{l}_{i}, \widetilde{q}_{R} \widetilde{q}_{R}} c_{q}^{2}\right) S\left(\hat{s} ; m_{\widetilde{q}_{2}}^{2}\right)\right] .
\end{array}
$$


These truncated amplitudes apply to the production of pairs of same-chirality sleptons of first and second generations, for any value of $\tan \beta$, and to the production of a pair of same-chirality $\widetilde{\tau}$ 's, for small values of $\tan \beta$.

When $\tan \beta$ is large, however, the chirality-mixing terms in the $\widetilde{\tau}$ sector may not be small. (See also Sec. 2.2.) Thus, $\widetilde{\tau}_{L}$ and $\widetilde{\tau}_{R}$ are not mass eigenstates anymore. The truncated amplitudes for the production of the three final states $\widetilde{\tau}_{1} \widetilde{\tau}_{1}, \widetilde{\tau}_{2} \widetilde{\tau}_{2}$, and $\widetilde{\tau}_{1} \widetilde{\tau}_{2}$ are

$$
\begin{aligned}
& \widetilde{A}_{\mathrm{QC}}^{\widetilde{\tau}_{1} \widetilde{\tau}_{1}}=c_{\tau}^{2} \widetilde{A}_{\mathrm{QC}}^{\widetilde{\tau}_{L} \widetilde{\tau}_{L}}+s_{\tau}^{2} \widetilde{A}_{\mathrm{QC}}^{\widetilde{\tau}_{R} \widetilde{R}_{R}}, \\
& \widetilde{A}_{\mathrm{QC}}^{\tilde{\tau}_{2} \tilde{\tau}_{2}}=s_{\tau}^{2} \widetilde{A}_{\mathrm{QC}}^{\widetilde{\tau}_{L} \tilde{\tau}_{L}}+c_{\tau}^{2} \widetilde{A}_{\mathrm{QC}}^{\widetilde{\tau}_{R} \tilde{\tau}_{R}}, \\
& \widetilde{A}_{\mathrm{QC}}^{\tilde{\tau}_{1} \widetilde{\tau}_{2}}=\left(s_{\tau} c_{\tau}\right)\left[\widetilde{A}_{\mathrm{QC}}^{\widetilde{\tau}_{L} \tilde{\tau}_{L}}-\widetilde{A}_{\mathrm{QC}}^{\widetilde{\tau}_{R} \tilde{\tau}_{R}}\right] .
\end{aligned}
$$

If among the nonvanishing terms of $V_{\text {ch-mix }}$ there is also $\left(\widetilde{m}_{D, R L}^{2}\right)_{3,3}$, F-terms induce a contribution to the cross section for the production of opposite-chirality sleptons. This is of relevance only for large values of $\tan \beta$ and for the production of a pair of $\widetilde{\tau}$ sleptons. Notice that no chirality-mixing terms in the $\widetilde{\tau}$ sector is necessary for this contribution to exist. As mentioned earlier, however, since $\tan \beta$ is large, it is hard to imagine that such mixing terms really vanish. The truncated amplitudes for the production of the three final states $\widetilde{\tau}_{1} \widetilde{\tau}_{1}, \widetilde{\tau}_{2} \widetilde{\tau}_{2}$, and $\widetilde{\tau}_{1} \widetilde{\tau}_{2}$ are now

$$
\begin{aligned}
& \widetilde{A}_{\mathrm{QC}}^{\widetilde{\tau}_{1} \widetilde{\tau}_{1}}=+2\left(s_{\tau} c_{\tau}\right) \widetilde{A}_{\mathrm{QC}}^{\tilde{\tau}_{L} \widetilde{\tau}_{R}}, \\
& \widetilde{A}_{\mathrm{QC}}^{\widetilde{\tau}_{2} \widetilde{\tau}_{2}}=-2\left(s_{\tau} c_{\tau}\right) \widetilde{A}_{\mathrm{QC}}^{\widetilde{\tau}_{L} \widetilde{\tau}_{R}}, \\
& \widetilde{A}_{\mathrm{QC}}^{\widetilde{\tau}_{1} \widetilde{\tau}_{2}}=-\left(c_{\tau}^{2}-s_{\tau}^{2}\right) \widetilde{A}_{\mathrm{QC}}^{\tau_{L}} . \widetilde{\tau}_{R} .
\end{aligned}
$$

Here, $\widetilde{A}_{\mathrm{QC}}^{\widetilde{\tau}_{L} \tilde{\tau}_{R}}$ is the truncated amplitude obtained from the F-term quartic couplings in the limit of vanishing chirality-mixing terms in the $\widetilde{\tau}$ sector:

$$
\widetilde{A}_{\mathrm{QC}}^{\widetilde{\tau}_{L} \tilde{\tau}_{R}}=-\frac{g_{2}^{2}}{2} \frac{m_{b} m_{\tau}}{M_{W}^{2}} \frac{1}{\cos ^{2} \beta}\left(s_{b} c_{b}\right)\left[S\left(\hat{s} ; \widetilde{m}_{b_{1}}^{2}\right)-S\left(\hat{s} ; \widetilde{m}_{b_{2}}^{2}\right)\right] .
$$

Notice that these truncated amplitudes scale as $\tan ^{2} \beta$, for any value of $\tan \beta \gtrsim 3$, and are also very sensitive to $\left|\left(\widetilde{m}_{D, \mathrm{RL}}^{2}\right)_{3,3}\right|$. Since $\left|\cos \theta_{b} \sin \theta_{b}\right| \sim\left|\left(\widetilde{m}_{D, \mathrm{RL}}^{2}\right)_{3,3}\right| /\left(\widetilde{m}_{b_{2}}^{2}-\widetilde{m}_{b_{1}}^{2}\right)$, they roughly scale linearly in this parameter.

At large $\tan \beta$, when both contributions from D- and F-term couplings exist, the truncated amplitudes $\widetilde{A}_{\mathrm{QC}}^{\widetilde{\mathrm{\tau}}_{1}}, \widetilde{A}_{\mathrm{QC}}^{\tilde{\tau}_{2} \tau_{2}}$, and $\widetilde{A}_{\mathrm{QC}}^{\tilde{\tau}_{1} \tau_{2}}$ are obtained by summing up those in Eqs. (14) and (15).

\subsection{Results}

We are now in a position to show some results, focussing on spectra that showcase separately the contribution from $\mathrm{F}$ - and D-terms. 


\subsubsection{D-term contributions}

For the first spectrum, or spectrum A, we choose all left-handed squarks and the righthanded ones of down-type to be heavy, i.e. $1 \mathrm{TeV}$, whereas the right-handed squarks of up-type and all sleptons are light. The values chosen here for the massive parameters in the down-squark sector are somewhat extreme, given how light the other squarks are. Indeed, it does not need to be so large, as the contribution from loops with squark heavier than a few hundred GeV decouples quite rapidly. The chirality-mixing terms $\left(\widetilde{m}_{U, \mathrm{RL}}^{2}\right)_{i i}$ and $\left(\widetilde{m}_{E, \mathrm{RL}}^{2}\right)_{i i}$, with $i=1,2,3$, are assumed to be vanishing, whereas the terms $\left(\widetilde{m}_{D, \mathrm{RL}}^{2}\right)_{i i}$ do not need to be specified, as the down-squarks are decoupled from our problem. Thus, the diagonal entries in the up-squark mass matrix and in the slepton mass matrices are mass eigenvalues. We denote the up-squarks relevant for our calculation $\widetilde{m}_{u_{R}}, \widetilde{m}_{c_{R}}, \widetilde{m}_{t_{R}}$. Under the assumption that all three entries in the diagonal matrix $\widetilde{m}_{\bar{U}}^{2}$ are equal, $\widetilde{m}_{u_{R}}$, $\widetilde{m}_{c_{R}}$ are given by $\widetilde{m}_{q}^{2} \simeq\left(\widetilde{m}_{\bar{U}}^{2}\right)_{i i}-M_{Z}^{2} / 6$, for $i=1,2$ (see Eq. (12)), whereas $\widetilde{m}_{t_{R}}$ receives also the F-term contribution $m_{t}^{2}$.

In summary, this spectrum is specified as follows:

$$
\begin{aligned}
& \underline{\text { spectrum A : }} \\
& \text { squarks : } \begin{cases}\left(\widetilde{m}_{Q}^{2}\right)_{i i}=\left(\widetilde{m}_{\bar{D}}^{2}\right)_{i i}=(1 \mathrm{TeV})^{2} & (i=1,2,3), \\
\left(\widetilde{m}_{U, \mathrm{RL}}^{2}\right)_{i i} \simeq 0 & (i=1,2,3), \\
\widetilde{m}_{u_{R}}^{2}=\widetilde{m}_{c_{R}}^{2} \equiv \widetilde{m}_{q}^{2} \ll\left(\widetilde{m}_{Q}^{2}\right)_{i i}, & \\
\widetilde{m}_{t_{R}}^{2}=\left(\widetilde{m}_{q}\right)^{2}+m_{t}^{2}, & \end{cases} \\
& \text { sleptons : } \quad\left\{\begin{array}{l}
\left(\widetilde{m}_{E, \mathrm{RL}}^{2}\right)_{i i} \simeq 0 \\
\widetilde{m}_{e_{L}}=\widetilde{m}_{\mu_{L}}=\widetilde{m}_{\tau_{L}}, \\
\widetilde{m}_{e_{R}}=\widetilde{m}_{\mu_{R}}=\widetilde{m}_{\tau_{R}} .
\end{array} \quad(i=1,2,3),\right.
\end{aligned}
$$

With this spectrum, only the production of same-chirality sleptons is possible, induced

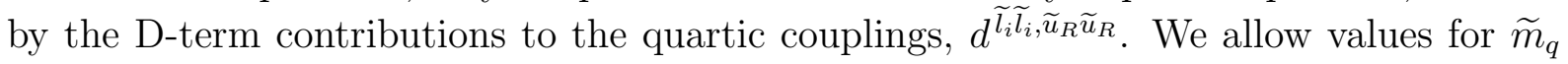
in the interval $[100,200] \mathrm{GeV}$. Notice that the mass of the third generation right-handed $\widetilde{t}$ squarks ranges approximately between 200 and $260 \mathrm{GeV}$. Since it is heavier, it gives a contribution to the cross sections smaller than that of the other right-handed up squarks.

The production cross sections are shown in Fig. 2 as a function of $\widetilde{m}_{q}$. The upper line (the red solid line) corresponds to the production of right-handed sleptons, which decreases from $1.6 \mathrm{fb}$ to $0.25 \mathrm{fb}$ in the mass range considered. The lower line (the blue dot-dashed line) corresponds to the production of left-handed sleptons, which is smaller by a factor four. This is due to the fact that the hypercharge of right-handed leptons is

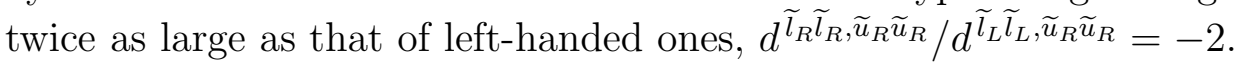

These, as well as all other cross sections shown in this paper are obtained using the CTEQ6L parton distribution functions [10], with factorization and renormalization scales fixed at $\sqrt{\hat{s}}$ and the adaptive Monte Carlo integration program BASES [11].

The cross sections shown in this figure apply to the production of first and second 


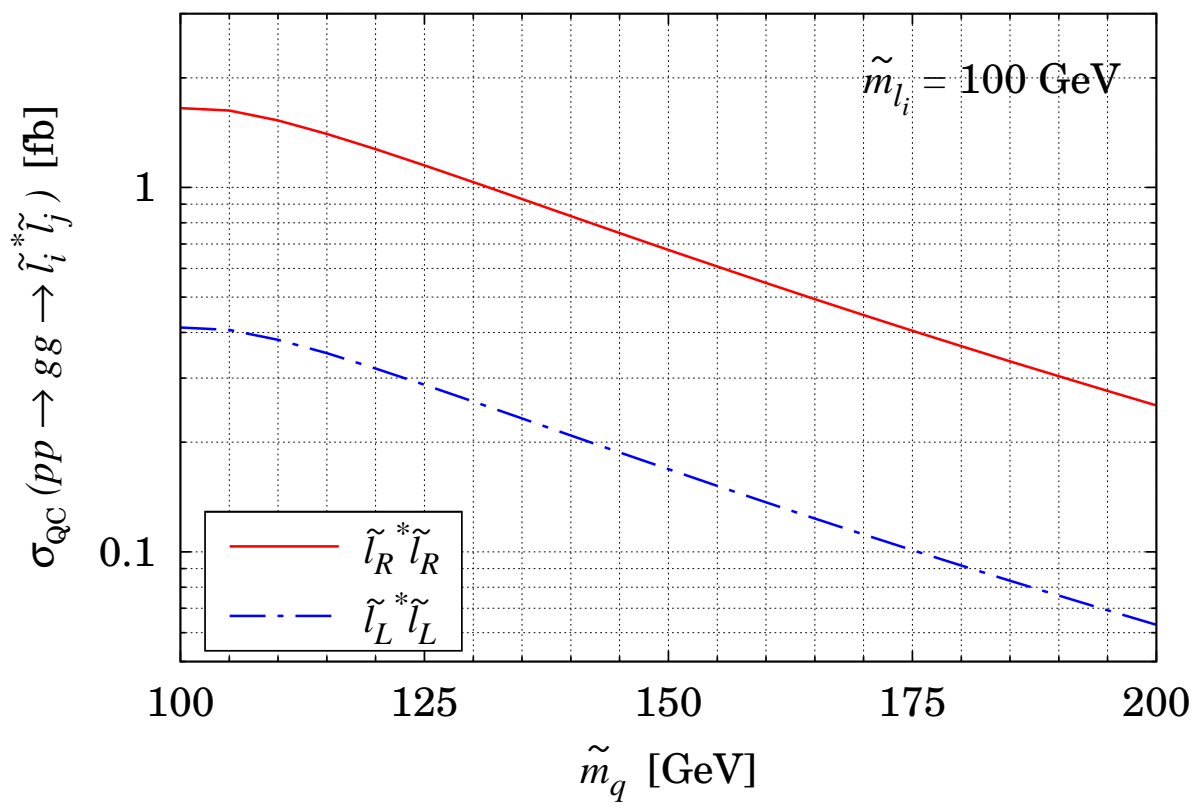

Figure 2: Quartic-coupling induced cross sections for the production of a pair of $100 \mathrm{GeV}$, samechirality sleptons obtained for the spectrum A (see Eq. (17)). The upper (red solid) and lower (blue dot-dashed) line correspond to right-handed and left-handed sleptons, respectively. These cross sections apply to the production of first and second generation sleptons for any value of $\tan \beta$, of third generation, only for low tan beta (see discussion in the text).

generation sleptons, for any value of $\tan \beta$, and to the production of a $\widetilde{\tau}$ 's pair only for low $\tan \beta$. This is due to the fact that for large values of $\tan \beta$ the Yukawa coupling of the $\tau$ lepton is nonnegligible, and the condition $\left(\widetilde{m}_{E, \mathrm{RL}}^{2}\right)_{33} \simeq 0$ can only be realized by tuning $\left(A_{E}\right)_{3,3}$ to cancel $\mu \tan \beta$. Large values of $\left|\left(A_{E}\right)_{3,3}\right|$, however, may have to be required for this cancellation, possibly producing charged minima lower than the electroweak one [7]. Of course it is still possible to assume that the local charge-preserving vacuum is metastable, with a lifetime greater than the age of the Universe [8]. Nevertheless, given how light the slepton spectrum is, also the metastability of the charge-preserving vacuum may be jeopardized by a large value of $\tan \beta$. Therefore, it is safe to say that the cross section in this figure apply to the production of a pair of $\widetilde{\tau}$ 's only in the case of small values of $\tan \beta$.

It should be noted here that the spectrum $\mathbf{A}$ has been tailored to maximize the value of these two production cross sections. On one side, the lightness of some of the squarks in this spectrum is extreme. On the other side, the choice of the up-type right-handed squarks as the light ones, has also contributed to increase the cross sections. The choice of the right-handed down squarks as light would have given smaller cross section, due to the fact that the corresponding quartic couplings have a ratio $d^{\widetilde{l}_{i} \widetilde{l}_{i}, \widetilde{u}_{R} \widetilde{u}_{R}} / d^{\widetilde{l}_{i} \widetilde{i}_{i}, \widetilde{d}_{R} \widetilde{d}_{R}}=-2$. A similar suppression factor is found in the situation in which the left-handed squarks are light, whereas the right-handed ones decouple from the problem. Due to $\mathrm{SU}(2)_{L}$ gauge 
invariance, the equality of the eigenvalues $\widetilde{m}_{u_{L}}^{2}=\widetilde{m}_{d_{L}}^{2}$ holds to a very good approximation, except for the third generation. Thus, the two squarks $\widetilde{u}_{L}$ and $\widetilde{d}_{L}$ exchanged in the loop contribute only with the $\mathrm{U}(1)_{Y}$ part of their couplings $d^{\widetilde{l}_{i} \widetilde{l}_{i}, \widetilde{u}_{L} \widetilde{u}_{L}}$ and $d^{\widetilde{l}_{i} \widetilde{l}_{i}, \widetilde{d}_{L} \widetilde{d}_{L}}$. As Table 1 shows, they contribute to the cross sections as much as one right-handed down squark of equal mass does.

The cross sections shown in this figure, therefore, can be considered as an upper bound for the value of quartic-coupling induced cross sections. One could try to increase this bound by taking $\left(\widetilde{m}_{Q}^{2}\right)_{33}$ smaller than $\left(\widetilde{m}_{Q}^{2}\right)_{i i}(i=1,2)$, and therefore lowering the value of $\widetilde{m}_{t_{R}}$. The gain is, however, quite small, as $\widetilde{m}_{t_{R}}$ cannot get down to the values of $\widetilde{m}_{u_{R}}$ and $\widetilde{m}_{c_{R}}$. A substantial chirality-mixing term in the $\widetilde{t}$ squark sector, could lower the value of $\widetilde{m}_{t_{1}}$. As Eq. (14) shows, the contribution of such a lighter $\widetilde{t}_{1}$ to the cross section would, however, get penalized by the mixing angle $\sin \theta_{t}$.

\subsubsection{F-term contributions}

Large chirality-mixing terms in the $\widetilde{b}$-squark sector are needed to have a nonvanishing contribution to the production of opposite-chirality $\widetilde{\tau}$ sleptons from the F-term $\left(h_{D}\right)_{33}\left(h_{E}\right)_{33}$. Indeed, the corresponding cross section scales slightly more strongly than $r_{b}^{2}$, where $r_{b}$ is defined as $\left|\left(\widetilde{m}_{D, \mathrm{RL}}^{2}\right)_{3,3}\right|=r_{b} \widetilde{m}_{b_{1}}^{2}$, and as $\left(\sin 2 \theta_{b}\right)^{2}$ (see Eq. (16)). Thus, both these parameters need to be maximized, while keeping the $\widetilde{b}$-squark and the $\widetilde{\tau}$-slepton relatively light, in order to explore an upper bound for this cross section. Moreover, given its scaling like $(\tan \beta)^{4}$, also $\tan \beta$ needs to be large. As a consequence, the $\widetilde{\tau}$-slepton sector needs to be strongly mixed as well.

We start by requiring $\cos \theta_{b}=\sin \theta_{b}=1 / \sqrt{2}$, so that $\cos 2 \theta_{b}=1$ (see Eq. (11)). This requirement fixes also $\widetilde{m}_{b_{2}}^{2}$ in terms of $\widetilde{m}_{b_{1}}^{2}$ and $r_{b}$. It is, indeed, $\widetilde{m}_{b_{2}}^{2}=\left(1+2 r_{b}\right) \widetilde{m}_{b_{1}}^{2}$. Strictly speaking, only the $\widetilde{b}$-squark sector needs to be specified for this cross section. Nevertheless, if we require also a maximal mixing in the $\widetilde{t}$ sector, 1.e. $\cos \theta_{t}=\sin \theta_{t}=1 / \sqrt{2}$, because of $\mathrm{SU}(2)_{L}$ gauge invariance, the above numbers specify also the diagonal entries in the $\tilde{t}$ squark mass matrix. As a consequence, $\widetilde{m}_{t_{1}}^{2}$ and $\widetilde{m}_{t_{2}}^{2}$ are fixed in terms of $\widetilde{m}_{b_{1}}^{2}, r_{b}$ and $r_{t}$, a parameter analogous to $r_{b}$ : $\left|\left(\widetilde{m}_{U, \mathrm{RL}}^{2}\right)_{3,3}\right|=r_{t} \widetilde{m}_{t_{1}}^{2}$. The choice $r_{t}=r_{b}$ gives a $\tilde{t}$-squark sector closely mirroring the $\widetilde{b}$-squark sector, and it helps suppressing the production cross section of same-chirality sleptons through a partial cancellation of the D-term contributions. By pushing all other squark masses at the TeV level, all other contributions from D-term couplings are suppressed.

We therefore examine the following squark spectrum:

$$
\begin{aligned}
& \left(\widetilde{m}_{Q}^{2}\right)_{i i}=\left(\widetilde{m}_{D}^{2}\right)_{i i}=\left(\widetilde{m}_{U}^{2}\right)_{i i}=(1 \mathrm{TeV})^{2} \quad(i=1,2), \\
& \left|\left(\widetilde{m}_{U, \mathrm{RL}}^{2}\right)_{i i}\right|,\left|\left(\widetilde{m}_{D, \mathrm{RL}}^{2}\right)_{i i}\right| \ll\left(\widetilde{m}_{Q}\right)_{i i} \quad(i=1,2), \\
& \left|\left(\widetilde{m}_{D, \mathrm{RL}}^{2}\right)_{3,3}\right|=r_{b} \widetilde{m}_{b_{1}}^{2},\left|\left(\widetilde{m}_{U, \mathrm{RL}}^{2}\right)_{3,3}\right|=r_{b} \widetilde{m}_{t_{1}}^{2}, \quad \theta_{b}=\theta_{t}=45^{0}, \\
& \widetilde{m}_{t_{1}}^{2}=\left[\widetilde{m}_{b_{1}}^{2}\left(1+r_{b}\right)+m_{t}^{2}-\left(\frac{3}{4}\right) M_{Z}^{2}\right] /\left[1+r_{b}\right] \\
& \widetilde{m}_{b_{2}}^{2}=\left(1+2 r_{b}\right) \widetilde{m}_{b_{1}}^{2}, \widetilde{m}_{t_{2}}^{2}=\left(1+2 r_{b}\right) \widetilde{m}_{t_{1}}^{2},
\end{aligned}
$$




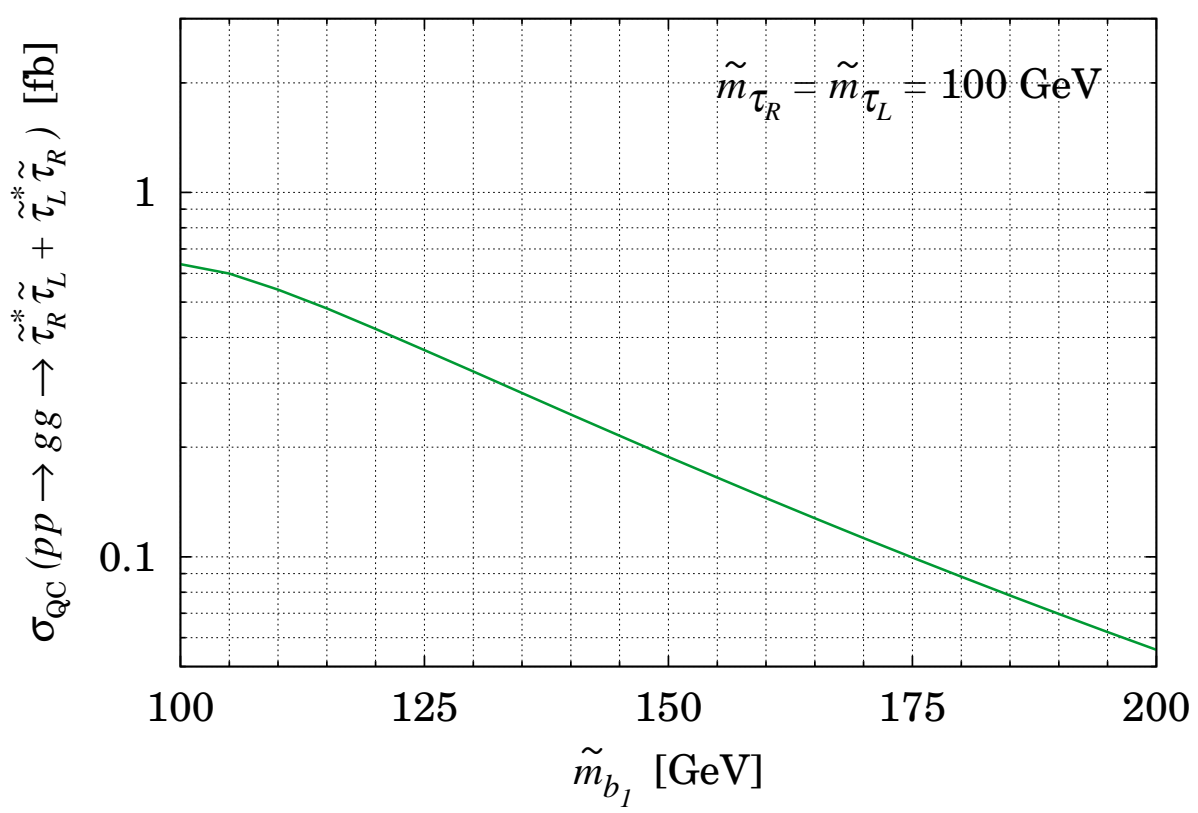

Figure 3: Cross section for the production of a pair of $100 \mathrm{GeV}$ opposite chirality $\widetilde{\tau}$ 's induced by F-term quartic-coupling, for $\tan \beta=30$ and the spectrum of Eq. (18). This may be regarded as an upper bound of the F-term contribution to the cross section; see discussion in the text.

If we let $\widetilde{m}_{b_{1}}$ take values in the same interval $[100,200] \mathrm{GeV}$ chosen before for the lightest squark masses in the spectrum $\mathbf{A}$, the values of $\widetilde{m}_{b_{2}}$ span an interval fixed by $r_{b}$, such as $[150,300] \mathrm{GeV}$ for $r_{b}=62.5 \%$. This choice brings $\left|\left(\widetilde{m}_{D, \mathrm{RL}}^{2}\right)_{3,3}\right|^{1 / 2}$ to be within $\sim 80$ and $160 \mathrm{GeV}$, the two $\widetilde{t}$ squark eigenstates $\widetilde{t}_{1}$ and $\widetilde{t}_{2}$ to have masses in the intervals $\sim[150,230] \mathrm{GeV}$ and $\sim[230,340] \mathrm{GeV}$, respectively, and $\left|\left(\widetilde{m}_{U, \mathrm{RL}}^{2}\right)_{3,3}\right|^{1 / 2}$ to lie between $\sim 120$ and $180 \mathrm{GeV}$.

We show in Fig. 3 the cross section for the production of opposite chirality $\widetilde{\tau}$ states of $100 \mathrm{GeV}$, assumed here to be mass eigenstates, obtained with this spectrum and $\tan \beta=$ 30. The cross section decreases from about $0.6 \mathrm{fb}$ to $0.06 \mathrm{fb}$ in this $\widetilde{b}_{1}$ mass range. Despite having a slightly steeper shape, it is comparable to the cross section for the production of a pair of left-handed sleptons shown in Fig. 2,

The values shown in this figure, however, may not be achieved for realistic sfermion spectra. The most serious problem is the magnitude of $\left|\left(A_{D}\right)_{33}\right|$, which tends to lie at the $1 \mathrm{TeV}$ level or above for all values of $r_{b}$, when $\tan \beta$ has the value chosen here. Such values of $\left|\left(A_{D}\right)_{33}\right|$ lead to a charge beaking vacuum [7], with lifetime shorter than the age of the Universe [8]. Towards the higher end of the values of $\widetilde{m}_{b_{1}}$ in Fig. 3, however, the value of $\left|\left(A_{D}\right)_{33}\right|$ can become sufficiently small to guarantee a long enough lifetime of the metastable electroweak vacuum, provided $\tan \beta$ does not significantly exceed the value of 10. An inspection of Fig. 33 shows that such a decrease in $\tan \beta$ would immediately plunge the corresponding cross section to values of $\mathcal{O}\left(10^{-2}\right) \mathrm{fb}$. An additional suppression comes also from relaxing the assumption of vanishing chirality-mixing terms in the $\widetilde{\tau}$-slepton 
sector, made for this figure. This assumption is also a problematic one, since it induces unacceptable values for $\left|\left(A_{E}\right)_{33}\right|$.

We therefore abondon the idea of studying further the contribution from F-term quartic couplings to the slepton production cross section from gluon fusion. Hereafter, the contribution from quartic couplings to the cross section should be understood as induced by D-terms only.

\section{The one-loop $g g \rightarrow \widetilde{l}^{*} \widetilde{l}$ cross section}

Effective $g g \widetilde{l}_{i}^{*} \widetilde{l}_{j}$ interactions can also be induced by the decay into a slepton pair of a neutral Higgs boson, produced at the one-loop level through gluon fusion. Examples of Feynmann diagrams giving rise to these effective interactions are shown in Fig. 4. There exists also a squark-bubble diagram analogous to that in Fig. 1, with a neutral Higgs boson emerging from the bubble to produce the slepton pair. The complete cross section including also the contribution from these diagrams has a more complicated dependence on the supersymmetric parameter space than that obtained only from slepton-squark quartic couplings, as it depends also on the Higgs-sector parameters.

The neutral Higgs boson exchanged in the s-channel is one of the mass eigenstates obtained from suitable rotations of the neutral components of the two Higgs doublets $H_{d}^{0}$ and $H_{u}^{0}$, the two scalar Higgs bosons, $h$ and $H$, and the pseudoscalar one, $A$ :

$$
\begin{aligned}
& H_{d}^{0}=\frac{1}{\sqrt{2}}\left\{v \cos \beta+(\cos \alpha H-\sin \alpha h)+i\left(\sin \beta A+\cos \beta \chi^{0}\right)\right\}, \\
& H_{u}^{0}=\frac{1}{\sqrt{2}}\left\{v \sin \beta+(\sin \alpha H+\cos \alpha h)+i\left(\cos \beta A-\sin \beta \chi^{0}\right)\right\},
\end{aligned}
$$

where $\chi^{0}$ is the Goldstone boson. Once radiative corrections to the Higgs sector are included, the angle $\alpha$ is determined by the relation [12]:

$$
\tan 2 \alpha=\frac{m_{A}^{2}+M_{Z}^{2}}{m_{A}^{2}-M_{Z}^{2}+\epsilon / \cos 2 \beta} \tan 2 \beta, \quad 0>\alpha>-\frac{\pi}{2},
$$

where $\epsilon$ is a correction factor proportional to the fourth power of the $t$-quark mass:

$$
\epsilon=\frac{3 G_{F}}{\sqrt{2} \pi^{2}} \frac{m_{t}^{4}}{\sin ^{2} \beta} \log \left(1+\frac{\widetilde{m}^{2}}{m_{t}^{2}}\right)
$$

with $\widetilde{m}$, the scale of the $\widetilde{t}$ scalar masses. The mass of the two physical states $h$ and $H$ are

$$
\begin{aligned}
m_{h}^{2} & \leq M_{Z}^{2}|\cos 2 \beta|+\epsilon \sin ^{2} \beta, \\
m_{H}^{2} & =m_{A}^{2}+M_{Z}^{2}-m_{h}^{2}+\epsilon,
\end{aligned}
$$

with the complete expression for $m_{h}$ given by:

$$
m_{h}^{2}=\frac{m_{A}^{2}+M_{Z}^{2}+\epsilon}{2}-\left[\left(\frac{m_{A}^{2}+M_{Z}^{2}+\epsilon}{2}\right)^{2}-m_{A}^{2} M_{Z}^{2} \cos ^{2} 2 \beta-\epsilon\left(m_{A}^{2} \sin ^{2} \beta+M_{Z}^{2} \cos ^{2} \beta\right)\right]^{1 / 2}
$$



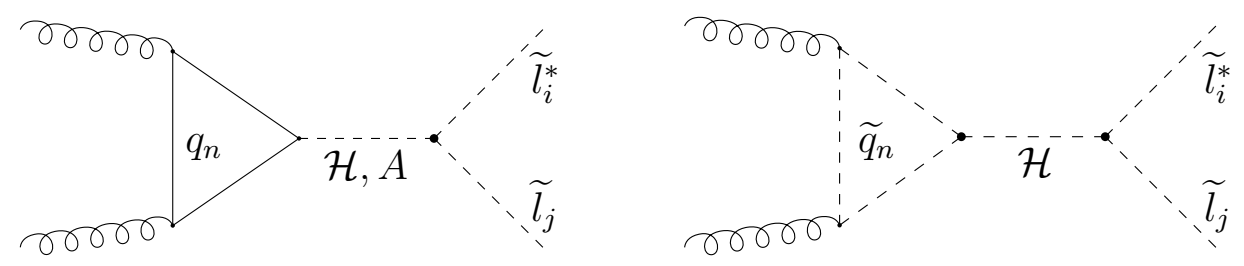

Figure 4: Diagrams contributing to the effective couplings gg $\widetilde{l}_{i}^{*} \widetilde{l}_{j}$, through exchange of a neutral Higgs boson in the s-channel. The symbol $\mathcal{H}$ denotes $h$ and $H$.

whereas $m_{A}$ can be considered a free parameter. Although the state-of-the-art of radiative corrections to the Higgs sector [13] is far more sophisticated than the sketchy picture given here, this description is sufficient for our discussion. In the following numerical evaluations, we use the Higgs boson masses and widths from CPSUPERH [14].

The three neutral Higgs bosons $h, H$, and $A$ couple to a fermion $f$ as:

$$
V_{\mathrm{H}-\mathrm{ferm}}=Y_{h f f} \bar{f} f h+Y_{H f f} \bar{f} f H+i Y_{A f f} \bar{f} \gamma_{5} f A+i Y_{\chi^{0} f f} \bar{f} \gamma_{5} f \chi^{0},
$$

with

$$
\begin{aligned}
Y_{h t t} & =\frac{\left(h_{U}\right)_{3,3}}{\sqrt{2}} \cos \alpha, & Y_{H t t} & =\frac{\left(h_{U}\right)_{3,3}}{\sqrt{2}} \sin \alpha, \\
Y_{A t t}= & \frac{\left(h_{U}\right)_{3,3}}{\sqrt{2}} \cos \beta, & Y_{\chi^{0} t t} & =-\frac{\left(h_{U}\right)_{3,3}}{\sqrt{2}} \sin \beta,
\end{aligned}
$$

when $f$ is the $t$ quark, and

$$
\begin{aligned}
Y_{h b b} & =-\frac{\left(h_{D}\right)_{3,3}}{\sqrt{2}} \sin \alpha, & Y_{H b b} & =\frac{\left(h_{D}\right)_{3,3}}{\sqrt{2}} \cos \alpha \\
Y_{A b b} & =\frac{\left(h_{D}\right)_{3,3}}{\sqrt{2}} \sin \beta, & Y_{\chi^{0} b b} & =\frac{\left(h_{D}\right)_{3,3}}{\sqrt{2}} \cos \beta,
\end{aligned}
$$

when it is the $b$ quark.

The trilinear couplings of $h, H$, and $A$ to squarks and sleptons originate from the trilinear soft SUSY-breaking terms, which can be read off from Eqs. (44) and (6) when $v_{i} / \sqrt{2}$ is replaced by $H_{i}, i=u, d$, and from the superpotential $\mu$ term:

$$
V_{\text {tril }}=t^{H_{i}^{0} \widetilde{s}_{R} \widetilde{s}_{L}} H_{i}^{0}\left(\widetilde{s}_{R}^{*} \widetilde{s}_{L}\right)+f_{\mu}^{H_{i}^{0 *} \widetilde{s}_{R} \widetilde{s}_{L}} H_{i}^{0 *}\left(\widetilde{s}_{R}^{*} \widetilde{s}_{L}\right)+\text { H.c. } \quad\left\{\begin{array}{l}
s=u, d, l \\
i=u, d
\end{array},\right.
$$

as well as from quartic couplings from D- and F-terms:

$$
\begin{aligned}
& V_{D} \supset d^{H_{i}^{0} H_{i}^{0} \widetilde{s}_{n} \widetilde{s}_{n}}\left(H_{i}^{0 *} H_{i}^{0}\right)\left(\widetilde{s}_{n}^{*} \widetilde{s}_{n}\right), \\
& V_{F} \supset f^{H_{i}^{0} H_{i}^{0} \widetilde{s}_{n} \widetilde{s}_{n}}\left(H_{i}^{0 *} H_{i}^{0}\right)\left(\widetilde{s}_{n}^{*} \widetilde{s}_{n}\right),
\end{aligned} \quad\left\{\begin{array}{l}
s_{n}=u_{L}, d_{L}, l_{L}, u_{R}, d_{R}, l_{R}, \\
i=u, d
\end{array},\right.
$$

in which one of the two neutral Higgs bosons acquires a vacuum expectation value. A list of these couplings is given in Table 2 . 


\begin{tabular}{|c|c|c|c|c|c|}
\hline Higgs(es) & $s_{m} s_{n}$ & $d^{H_{i}^{0} H_{i}^{0}, \widetilde{s}_{n} \widetilde{s}_{n}}$ & $f^{H_{i}^{0} H_{i}^{0}, \widetilde{s}_{n} \widetilde{s}_{n}}$ & $f_{\mu}^{H_{i}^{0 *}, \widetilde{s}_{m} \widetilde{s}_{n}}$ & $t^{H_{i}^{0}, \widetilde{s}_{m} \widetilde{s}_{n}}$ \\
\hline$H_{d}^{0 *} H_{d}^{0}$ & $\widetilde{u}_{L}^{*} \widetilde{u}_{L}$ & $+\frac{g_{2}^{2}}{4}-\frac{g_{1}^{2}}{12}$ & - & - & - \\
\hline$H_{d}^{0 *} H_{d}^{0}$ & $\widetilde{d}_{L}^{*} \widetilde{d}_{L}$ & $-\frac{g_{2}^{2}}{4}-\frac{g_{1}^{2}}{12}$ & $h_{D}^{2}$ & - & - \\
\hline$H_{d}^{0 *} H_{d}^{0}$ & $\widetilde{u}_{R}^{*} \widetilde{u}_{R}$ & $+\frac{g_{1}^{2}}{3}$ & - & - & - \\
\hline$H_{d}^{0 *} H_{d}^{0}$ & $\widetilde{d}_{R}^{*} \widetilde{d}_{R}$ & $-\frac{g_{1}^{2}}{6}$ & $h_{D}^{2}$ & - & - \\
\hline$H_{d}^{0 *}$ & $\widetilde{t}_{R}^{*} \widetilde{t}_{L}$ & - & - & $-\mu\left(h_{U}\right)_{3,3}$ & - \\
\hline$H_{d}^{0}$ & $\widetilde{b}_{R}^{*} \widetilde{b}_{L}$ & - & - & - & $\left(A_{D}\right)_{3,3}\left(h_{D}\right)_{3,3}$ \\
\hline$H_{d}^{0 *} H_{d}^{0}$ & $\widetilde{l}_{L}^{*} \widetilde{l}_{L}$ & $-\frac{g_{2}^{2}}{4}+\frac{g_{1}^{2}}{4}$ & $h_{E}^{2}$ & - & - \\
\hline$H_{d}^{0 *} H_{d}^{0}$ & $\widetilde{l}_{R}^{*} \widetilde{l}_{R}$ & $-\frac{g_{1}^{2}}{2}$ & $h_{E}^{2}$ & - & - \\
\hline$H_{d}^{0}$ & $\widetilde{\tau}_{R}^{*} \widetilde{\tau}_{L}$ & - & - & - & $\left(A_{E}\right)_{3,3}\left(h_{E}\right)_{3,3}$ \\
\hline$H_{u}^{0 *} H_{u}^{0}$ & $\widetilde{u}_{L}^{*} \widetilde{u}_{L}$ & $-\frac{g_{2}^{2}}{4}+\frac{g_{1}^{2}}{12}$ & $h_{U}^{2}$ & - & - \\
\hline$H_{u}^{0 *} H_{u}^{0}$ & $\widetilde{d}_{L}^{*} \widetilde{d}_{L}$ & $+\frac{g_{2}^{2}}{4}+\frac{g_{1}^{2}}{12}$ & - & - & - \\
\hline$H_{u}^{0 *} H_{u}^{0}$ & $\widetilde{u}_{R}^{*} \widetilde{u}_{R}$ & $-\frac{g_{1}^{2}}{3}$ & $h_{U}^{2}$ & - & - \\
\hline$H_{u}^{0 *} H_{u}^{0}$ & $\widetilde{d}_{R}^{*} \widetilde{d}_{R}$ & $+\frac{g_{1}^{2}}{6}$ & - & - & - \\
\hline$H_{u}^{0}$ & $\tilde{t}_{R}^{*} \widetilde{t}_{L}$ & - & - & - & $\left(A_{U}\right)_{3,3}\left(h_{U}\right)_{3,3}$ \\
\hline$H_{u}^{0 *}$ & $\widetilde{b}_{R}^{*} \widetilde{b}_{L}$ & - & - & $-\mu\left(h_{D}\right)_{3,3}$ & - \\
\hline$H_{u}^{0 *} H_{u}^{0}$ & $\widetilde{l}_{L}^{*} \widetilde{l}_{L}$ & $+\frac{g_{2}^{2}}{4}-\frac{g_{1}^{2}}{4}$ & - & - & - \\
\hline$H_{u}^{0 *} H_{u}^{0}$ & $\widetilde{l}_{R}^{*} \widetilde{l}_{R}$ & $+\frac{g_{1}^{2}}{2}$ & - & - & - \\
\hline$H_{u}^{0 *}$ & $\widetilde{\tau}_{R}^{*} \widetilde{\tau}_{L}$ & - & - & $-\mu\left(h_{E}\right)_{3,3}$ & - \\
\hline
\end{tabular}

Table 2: Scalar potential couplings of the interactions among the neutral components of the two Higgs doublets and sfermions. The Hermitian conjugates of the interactions listed here between one Higgs boson and two opposite-chirality sfermions also exist. In the approximation of real SUSY-breaking parameters and real $\mu$ their couplings coincide with those given in this table. 


\begin{tabular}{|c|c|c|}
\hline & $s_{n}=l_{L}, l_{R}\left(h_{S}=h_{E}\right) ; d_{L}, d_{R}\left(h_{S}=h_{D}\right)$ & $s_{n}=u_{L}, u_{R}$ \\
\hline$g_{h \widetilde{s}_{n} \widetilde{s}_{n}}$ & {$\left[s_{(\beta+\alpha)} d^{H_{u}^{0} H_{u}^{0} s_{n} s_{n}}-h_{S}^{2} c_{\beta} s_{\alpha}\right]$} & {$\left[s_{(\beta+\alpha)} d^{H_{u}^{0} H_{w}^{0} s_{n} s_{n}}+h_{U}^{2} s_{\beta} c_{\alpha}\right]$} \\
\hline \multirow[t]{2}{*}{$g_{H \widetilde{s}_{n} \widetilde{s}_{n}}$} & {$\left[c_{(\beta+\alpha)} d^{H_{d}^{0} H_{d}^{0}, s_{n} s_{n}}+h_{S}^{2} c_{\beta} c_{\alpha}\right]$} & {$\left[c_{(\beta+\alpha)} d^{H_{d}^{0} H_{d}^{0}, s_{n} s_{n}}+h_{U}^{2} s_{\beta} s_{\alpha}\right]$} \\
\hline & $s=l\left(h_{S}=h_{E}, A_{S}=A_{E}\right) ; d\left(h_{S}=h_{D}, A_{S}=A_{D}\right)$ & $s=u$ \\
\hline$g_{h \widetilde{s}_{R} \widetilde{s}_{L}}$ & $\left(-A_{S} s_{\alpha}-\mu c_{\alpha}\right) h_{S} /(\sqrt{2} v)$ & $\left(+A_{U} c_{\alpha}+\mu s_{\alpha}\right) h_{U} /(\sqrt{2} v)$ \\
\hline$g_{H \widetilde{s}_{R} \widetilde{s}_{L}}$ & $\left(+A_{S} c_{\alpha}-\mu s_{\alpha}\right) h_{S} /(\sqrt{2} v)$ & $\left(+A_{U} s_{\alpha}-\mu c_{\alpha}\right) h_{U} /(\sqrt{2} v)$ \\
\hline$g_{A \widetilde{s}_{R} \widetilde{s}_{L}}$ & $\left(+A_{S} s_{\beta}+\mu c_{\beta}\right) h_{S} /(\sqrt{2} v)$ & $\left(+A_{U} c_{\beta}+\mu s_{\beta}\right) h_{U} /(\sqrt{2} v)$ \\
\hline$g_{\chi^{0} \widetilde{s}_{R} \widetilde{s}_{L}}$ & $\left(+A_{S} c_{\beta}-\mu s_{\beta}\right) h_{S} /(\sqrt{2} v)$ & $\left(-A_{U} s_{\beta}+\mu c_{\beta}\right) h_{U} /(\sqrt{2} v)$ \\
\hline
\end{tabular}

Table 3: Couplings of sfermions to the mass eigenstate neutral Higgs bosons $\mathcal{H}=\{h, H\}$ and A. The couplings $g_{\mathcal{H} \widetilde{s}_{L} \widetilde{s}_{R}}, g_{A \widetilde{s}_{L} \widetilde{s}_{R}}$, and $g_{\chi \widetilde{s}_{L} \widetilde{s}_{R}}$, not explicitly listed, coincide respectively with

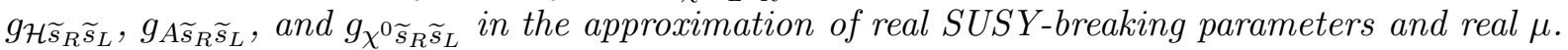
The symbols $c_{x}, s_{x}$ are abbreviations of $\cos x, \sin x$. Only the third generation Yukawa couplings $\left(h_{U}\right)_{3,3},\left(h_{D}\right)_{3,3}$, and $\left(h_{E}\right)_{3,3}$ are assumed to be nonvanishing.

In terms of the neutral mass eigenstate Higgs bosons, these trilinear interactions have the compact form:

$$
\begin{aligned}
V_{\mathrm{H}-\text { sfermion }} \supset & v\left(g_{h \widetilde{s}_{n} \widetilde{s}_{n}} h+g_{H \widetilde{s}_{n} \widetilde{s}_{n}} H\right)\left(\widetilde{s}_{n}^{*} \widetilde{s}_{n}\right)+ \\
& {\left[v\left(g_{h \widetilde{s}_{R} \widetilde{s}_{L}} h+g_{H \widetilde{s}_{R} \widetilde{s}_{L}} H+i g_{A \widetilde{s}_{R} \widetilde{s}_{L}} A+i g_{\chi} \widetilde{s}_{R} \widetilde{s}_{L}\right)\left(\widetilde{s}_{R}^{*} \widetilde{s}_{L}\right)+\text { H.c. }\right], }
\end{aligned}
$$

with couplings listed explicitly in Table 3 .

We note here that also in this case sum rules analogous to those in Eq. (10) exist for each generation of squarks:

$$
\sum_{\widetilde{q}_{n}} d^{H_{u}^{0} H_{w}^{0} \widetilde{q}_{n} \widetilde{q}_{n}}=\sum_{\widetilde{q}_{n}} d^{H_{d}^{0} H_{d}^{0} \widetilde{q}_{n} \widetilde{q}_{n}}=0 .
$$

As a consequence, similar sum rules hold for the D-terms contributions to the couplings $g_{h \widetilde{q}_{n} \widetilde{q}_{n}}$ and $g_{H \widetilde{q}_{n} \widetilde{q}_{n}}$.

This mechanism of production of a pair of sleptons through gluon fusion may also be regarded as a test of SUSY, although triple couplings of partners of the SM particles are not unique of SUSY models. In particular, unlike the Drell-Yan production mechanism, the gluon-fusion mechanism mediated by the exchange of a Higgs boson can probe the Higgs sector, the $\mu$ parameter, $\tan \beta$, and the trilinear $A$ terms.

The parton-level cross section for the gluon-fusion production of a pair of sleptons, can be expressed in terms of two form factors, which parametrize the effective Lagrangian 
induced at the one-loop level by quartic-couplings and Higgs-exchange diagrams:

$$
\mathcal{L}_{g g \rightarrow \widetilde{l}_{i}^{*} \widetilde{l}_{j}}=\frac{\alpha_{s}}{4 \pi}\left\{A_{S}^{\widetilde{l}_{i} \widetilde{l}_{j}}\left(g^{\mu \nu}-\frac{2 k_{2}^{\mu} k_{1}^{\nu}}{\hat{s}}\right)+A_{P}^{\widetilde{l}_{i} \widetilde{l}_{j}} \epsilon_{\alpha \beta}^{\mu \nu}\left(\frac{2 k_{1} k_{2}}{\hat{s}}\right)\right\} \delta_{a b} \epsilon_{\mu}^{\alpha}\left(k_{1}\right) \epsilon_{\nu}^{\beta}\left(k_{2}\right) \widetilde{l}_{i}^{*} \widetilde{l}_{j} .
$$

Here $\epsilon_{\mu \nu \alpha \beta}$ is a fully antisymmetric tensor, with $\epsilon_{0123}=1, k_{1}$ and $k_{2}$ are the momenta of the two initial gluons, and $A_{P}^{l_{i} l_{j}}$ is generated by the diagram with exchange of the pseudoscalar Higgs boson $A$, whereas all the other contributions are included in $A_{S}^{\widetilde{l}_{i} \widetilde{l}_{j}}$ :

$$
A_{S}^{\tilde{l}_{i} \widetilde{l}_{j}}=\widetilde{A}_{\mathrm{QC}}^{\widetilde{l}_{i} \widetilde{l}_{j}}+A_{S, \mathcal{H}}^{\tilde{l}_{i} \widetilde{l}_{j}}+\widetilde{A}_{S, \mathcal{H}}^{\widetilde{l}_{l} \tilde{l}_{j}}
$$

In this expression, the tilde distinguishes the contributions from scalar loops $\left(\widetilde{A}_{\mathrm{QC}}^{\tilde{l}_{i} \widetilde{l}_{j}}\right.$ is the truncated amplitude discussed in the previous section), and the subscript $\{\mathcal{H}\}$ refers to diagrams with exchange of a scalar Higgs boson, i.e. $\mathcal{H}=h, H$. Notice that, because of our assumption of imaginary $i g_{A \widetilde{s}_{L} \widetilde{s}_{R}}$ couplings and real chirality-mixing terms in the sfermion sector, there is no contribution to $A_{S}^{\tilde{l}_{i} \widetilde{l}_{j}}$ from the exchange of the pseudoscalar Higgs boson.

In terms of $A_{S}^{\widetilde{l}_{i} \widetilde{l}_{j}}$ and $A_{P}^{\widetilde{l}_{i} \widetilde{l}_{j}}$ the parton-level cross section is

$$
\widehat{\sigma}\left(g g \rightarrow \widetilde{l}_{i}^{*} \widetilde{l}_{j}\right)=\frac{\alpha_{s}^{2}}{(16 \pi)^{3}} \frac{\lambda^{1 / 2}\left(\hat{s}, \widetilde{m}_{l_{i}}, \widetilde{m}_{l_{j}}\right)}{\hat{s}}\left\{\left|A_{S}^{\widetilde{l}_{i} \widetilde{l}_{j}}\right|^{2}+\left|A_{P}^{\widetilde{l}_{i} \widetilde{l}_{j}}\right|^{2}\right\}
$$

The expression for $A_{S, \mathcal{H}}^{\tilde{l}_{i} \widetilde{l}_{j}}$ and $A_{P}^{\tilde{l}_{i} \widetilde{l}_{j}}$ (which strictly speaking should be labelled $A_{P, A} \tilde{l}_{i} \widetilde{l}_{j}$ ) coming from fermion loops are

$$
\begin{aligned}
A_{S, \mathcal{H}}^{\widetilde{l}_{i} \widetilde{l}_{i}} & =-\sum_{\mathcal{H}=h, H} g_{\mathcal{H} \widetilde{l}_{i} \widetilde{l}_{j}}\left(\frac{v}{m_{t}}\right)\left(\frac{1}{1-m_{\mathcal{H}}^{2} / \hat{s}+i m_{\mathcal{H}} \Gamma_{\mathcal{H}} / \hat{s}}\right) Y_{\mathcal{H} t t} F\left(\hat{s} ; m_{t}^{2}\right), \\
A_{P}^{\widetilde{l}_{L} \widetilde{l}_{R}} & =-g_{\mathcal{A} \widetilde{l}_{L} \widetilde{l}_{R}}\left(\frac{v}{m_{t}}\right)\left(\frac{1}{1-m_{A}^{2} / \hat{s}+i m_{A} \Gamma_{A} / \hat{s}}\right) Y_{A t t} P\left(\hat{s} ; m_{t}^{2}\right),
\end{aligned}
$$

with $A_{P}^{\widetilde{l}_{L} \widetilde{l}_{R}}=A_{P}^{\widetilde{l}_{R} \widetilde{l}_{L}}$ and $A_{P}^{\widetilde{l}_{L} \widetilde{l}_{L}}=A_{P}^{\widetilde{l}_{R} \widetilde{l}_{R}}=0$, that for $\widetilde{A}_{S, \mathcal{H}}^{\widetilde{l}_{\tilde{l}} \widetilde{l}_{i}}$ is

$$
\begin{aligned}
& \widetilde{A}_{S, \mathcal{H}}^{\tilde{l}_{i} \widetilde{l}_{i}}=-\sum_{\mathcal{H}=h, H} g_{\mathcal{H} \widetilde{l}_{i} \widetilde{l}_{i}}\left(\frac{v^{2}}{\hat{s}}\right)\left(\frac{1}{1-m_{\mathcal{H}}^{2} / \hat{s}+i m_{\mathcal{H}} \Gamma_{\mathcal{H}} / \hat{s}}\right) \times \\
& \sum_{q=u, d}\left[\left(c_{q}^{2} g_{\mathcal{H} \widetilde{q}_{L} \widetilde{q}_{L}}+s_{q}^{2} g_{\mathcal{H} \widetilde{q}_{R} \widetilde{q}_{R}}+2\left(s_{q} c_{q}\right) g_{\mathcal{H} \widetilde{q}_{R} \widetilde{q}_{L}}\right) S\left(\hat{s} ; \widetilde{m}_{q_{1}}^{2}\right)+\right. \\
& \left.\left(s_{q}^{2} g_{\mathcal{H} \widetilde{q}_{L} \widetilde{q}_{L}}+c_{q}^{2} g_{\mathcal{H} \widetilde{q}_{R} \widetilde{q}_{R}}-2\left(s_{q} c_{q}\right) g_{\mathcal{H} \widetilde{q}_{R} \widetilde{q}_{L}}\right) S\left(\hat{s} ; \widetilde{m}_{q_{2}}^{2}\right)\right],
\end{aligned}
$$

with $c_{q}=\cos q$ and $s_{q}=\sin q$, and $q$ spanning over all three generations. We remind that in the limit of vanishing chirality-mixing terms in the squark sector, $\cos q \rightarrow 0, \sin q \rightarrow 1$, and $\widetilde{q}_{1} \rightarrow \widetilde{q}_{R}, \widetilde{q}_{2} \rightarrow \widetilde{q}_{L}$. 


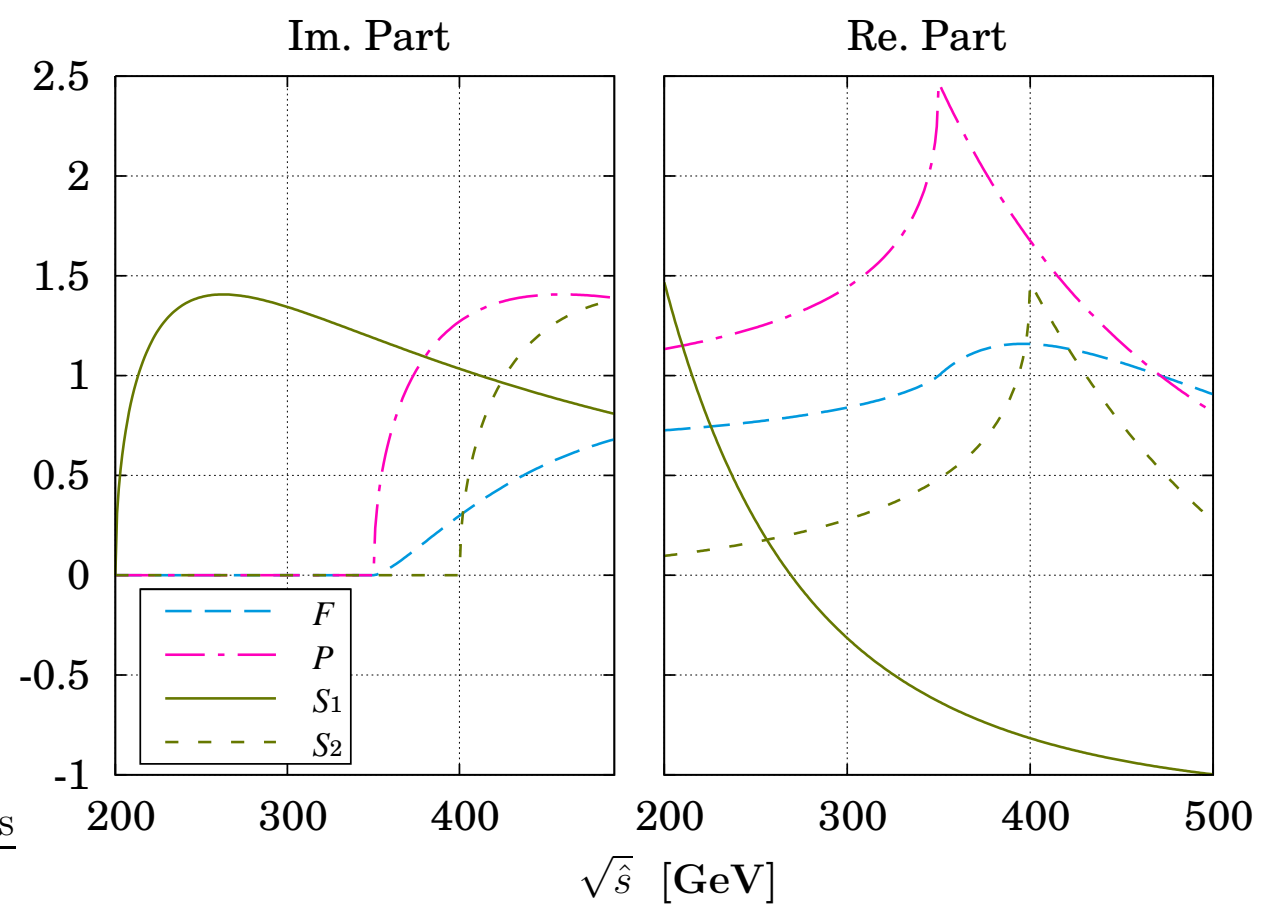

Figure 5: Imaginary and real part of the functions $F\left(\hat{s} ; m^{2}\right), P\left(\hat{s} ; m^{2}\right)$, and $S\left(\hat{s} ; m^{2}\right)$ versus $\hat{s}$. The value of $m$ is fixed to be $m_{t}$ for $F$ and $P$, shown by the azure long-dashed line and the magenta dot-dashed one, respectively. Two values of $m$ are chosen for $S$, shown by the two olive lines: $m=100 \mathrm{GeV}$ (solid line $S_{1}$ ), and $m=200 \mathrm{GeV}$ (short-dashed line $S_{2}$ ).

The function $S\left(\hat{s} ; m_{\widetilde{q}_{n}}^{2}\right)$ is given in Eq. (8) in Sec. 2.1, $F\left(\hat{s} ; m_{t}^{2}\right)$ and $P\left(\hat{s} ; m_{t}^{2}\right)$ are

$$
\begin{array}{ll}
F\left(\hat{s} ; m^{2}\right)=\tau[1+(1-\tau) f(\tau)], & \\
P\left(\hat{s} ; m^{2}\right)=\tau f(\tau), & \tau \equiv 4 m^{2} / \hat{s},
\end{array}
$$

with $f(\tau)$ already defined in Eq. (9). The imaginary and real parts of the three functions $F\left(\hat{s} ; m^{2}\right), P\left(\hat{s} ; m^{2}\right)$, and $S\left(\hat{s} ; m^{2}\right)$ are plotted in Fig. 5 versus $\hat{s}$. We have taken $m=m_{t}$ for $F$ and $P$, and the two values $m=100$ and $200 \mathrm{GeV}$ for $S$. The two resulting functions of $\hat{s}$ in this last case are called $S_{1}$ and $S_{2}$ in this figure. See the two olive lines, solid for $S_{1}$ and short-dashed for $S_{2}$. The azure long-dashed line and the red dot-dashed one represent $F$ and $P$, respectively.

It should be noted here that, because of the sum rules in Eqs. (10) and (30), the constant term -1 in the definition of the function $S$ does not contribute to the evaluation of $\widetilde{A}_{\mathrm{QC}}^{l_{i} l_{j}}$ and $\widetilde{A}_{S, \mathcal{H}}$, when the quartic and trilinear couplings considered here are determined by D-terms. In this case, the function $S$ contributes to these truncated amplitudes exactly like the function $P$. The values of the relevant real part of $S$ is then obtained shifting upward by the constant value 1 the two olive lines in Fig. 5 .

The functions $F$ and $P$ (and $S$, when the relevant couplings come from D-terms) vanish in the limit $\hat{s} \rightarrow \infty$, for fixed $m$, and also in the limit $m \rightarrow 0$, for fixed $\hat{s}$. For $m=m_{b}$, both the real and imaginary part of $F$ and $P$ are at most of $\mathcal{O}\left(10^{-3}\right)$. Nevertheless, the 
contribution from a bottom-quark loop, with truncated amplitudes analogous to those from the top quark given in Eqs. (34) and (35), is enhanced by the factor $\left(v / m_{b}\right)$, and unsuppressed values of the couplings $Y_{H b b}$ and $Y_{A b b}$ in the case of large $\tan \beta$. Depending on the value of $\tan \beta$, it may end up being the dominant contribution.

The generalization of the formulae in Eqs. (34), (35), and (36) to the case with nonvanishing chirality-mixing terms in the slepton sector can be easily obtained using the algorithm of Eq. (14) applied to $A_{S}^{l_{i} l_{j}}$ and $A_{P}^{l_{i} l_{j}}$.

\subsection{Results}

Even before presenting numerical results, it is possible to draw some general observations on the truncated amplitudes induced by the Higgs-boson exchange from an inspection of the couplings $Y_{\mathcal{H} t t}, Y_{A t t}, g_{\mathcal{H} \widetilde{q}_{i} \widetilde{q}_{j}}$, and $g_{A \widetilde{q}_{i} \widetilde{q}_{j}}$, as well as $g_{\mathcal{H} \widetilde{l}_{i} \widetilde{l}_{j}}$, and $g_{A \widetilde{l}_{i} \widetilde{l}_{j}}$, for a given Higgs spectrum.

We shall examine below three different Higgs spectra. Spectrum $\mathbf{I}$ has all three Higgs bosons, $h, H$, and $A$, light and maximally mixed, i.e. $\cos \alpha \sim-\sin \alpha \sim 1 / \sqrt{2}$. All the three states have masses below the threshold $\sqrt{\hat{s}_{\min }}=2 \widetilde{m}_{l_{i}}$. Thus, no resonance is encountered when integrating $\hat{s}$ from $\hat{s}_{\text {min }}$ to s. Spectrum II has $m_{A} \gtrsim 2 \widetilde{m}_{l}$, i.e. $m_{A} \gtrsim 200 \mathrm{GeV}$ in our case study; $m_{h}$ and $m_{H}$ are calculated as explained at the beginning of this section, and depend on $\tan \beta$ and the specific squark sector chosen. In this case, it is in general $\cos \alpha \sim 1$, and $\sin \alpha$ is small, but nonnegligible. The resonant contributions of $H$ and $A$ are expected to lift up the values of our cross sections. Spectrum III has $A$ and $H$ rather heavy and completely decoupled from our problem. The mixing angle $\alpha$ is such that $\cos \alpha=1$ and $\sin \alpha=0$, to a very good accuracy.

We consider first the case of vanishing chirality-mixing terms in the squark sector, as in the squark spectrum $\mathbf{A}$ specified in the previous section. In this context we start concentrating on the couplings $g_{\mathcal{H} \widetilde{q}_{L} \widetilde{q}_{L}}$ and $g_{\mathcal{H} \widetilde{q}_{R} \widetilde{q}_{R}}$, the only ones giving a nonvanishing contribution to $\widetilde{A}_{S, \mathcal{H}}^{\tilde{l}_{\tilde{l}}}$.

Because of the sum rule in Eq. (30), also the contribution to $\widetilde{A}_{S, \mathcal{H}}^{\widetilde{l}_{i} \widetilde{l}_{i}}$ from D-terms, as the contribution to $\widetilde{A}_{\mathrm{QC}}^{\tilde{l}_{i} \widetilde{l}_{i}}$, vanishes identically in the limit of a completely degenerate squark spectrum. The squark spectrum $\mathbf{A}$, which we shall use in the following, was however conceived precisely with the aim of evading the effectiveness of this sum rule.

Unlike in the case of $\widetilde{A}_{\mathrm{QC}}^{\widetilde{l}_{i} \widetilde{\tau}_{i}}, \mathrm{~F}$-terms give now contributions to $\widetilde{A}_{S, \mathcal{H}}^{\widetilde{l}_{i} \widetilde{\tau}_{i}}$, that are nonvanishing. By inspecting the couplings $g_{\mathcal{H}} \widetilde{q}_{L} \widetilde{q}_{L}$ and $g_{\mathcal{H} \widetilde{q}_{R} \widetilde{q}_{R}}$ in Table 3, it is easy to see that F-terms assign a privileged role to the $\widetilde{t}$ squarks. All other F-term contributions to these couplings are small even for large values of $\tan \beta$. This holds in particular also for the $\widetilde{b}$ squarks, whose couplings receive an F-term contribution always multiplied by $\cos \beta$.

In addition, the F-term contributions to the couplings $g_{\mathcal{H} \widetilde{t}_{L} \widetilde{t}_{L}}$ and $g_{\mathcal{H}_{t_{R}} \widetilde{t}_{R}}$ are in general larger than the contributions from D-terms to any of the couplings $g_{\mathcal{H} \widetilde{q}_{L} \widetilde{q}_{L}}$ and $g_{\mathcal{H} \widetilde{q}_{R} \widetilde{q}_{R}}$, for any $q$. In the case of the $h$ exchange, when, however, the cross section is expected to be 
small, $\left(h_{U}\right)_{33}^{2} \sin \beta \cos \alpha$ dominates over $\left|\sin (\beta+\alpha) d^{H_{u}^{0} H_{w}^{0} \widetilde{t}_{R} \widetilde{t}_{R}}\right|$ and $\left|\sin (\beta+\alpha) d^{H_{u}^{0} H_{w}^{0} \widetilde{t}_{L} \widetilde{t}_{L}}\right|$ by a considerable margin. Similarly, $\left|\left(h_{U}\right)_{33}^{2} \sin \beta \sin \alpha\right|$ dominates also over $\mid \cos (\beta+$ $\alpha) d^{H_{d}^{0} H_{d}^{0} \widetilde{t}_{R} \widetilde{t}_{R}}$ and $\left|\cos (\beta+\alpha) d^{H_{d}^{0} H_{d}^{0}, \widetilde{t}_{L} \widetilde{t}_{L}}\right|$, in the case of the heavier Higgs boson exchange $H$, except when $\sin \alpha$ is very close to its typical decoupling-limit value, $\sin \alpha=0$. In this limit, however, $H$ is too heavy to be of any relevance for our cross sections.

We notice also that, $\left|g_{\mathcal{H} \widetilde{t}_{L} \widetilde{t}_{L}}\right|,\left|g_{\mathcal{H} \widetilde{t}_{R} \widetilde{t}_{R}}\right| \gtrsim\left|Y_{\mathcal{H} t t}\right|$. Therefore a comparison of the relative size of $A_{S, \mathcal{H}}^{\tilde{l}_{l_{l}}}$ and $\widetilde{A}_{S, \mathcal{H}}^{\tilde{l}_{i}} \widetilde{l}_{i}$, amounts practically to a comparison of $\left(v / m_{t}\right) F\left(\hat{s}, m_{t}^{2}\right)$ versus $\sum_{i}\left(v^{2} / \hat{s}\right) S\left(\hat{s}, \widetilde{m}_{t_{i}}^{2}\right), i=1,2$. The $\tilde{t}$ squark loop is clearly penalized by a factor $\left(v^{2} / \hat{s}\right)$ with respect to the factor $\left(v / m_{t}\right)$, constant in $\hat{s}$. Moreover, as Fig. 5 shows, when integrated in $\hat{s}$, the function $S$ gives a smaller contribution to $\widetilde{A}_{S, \mathcal{H}}^{\tilde{l}_{l_{i}}}$ than the function $F$ gives to $A_{S, \mathcal{H}}^{\widetilde{l}_{i} \widetilde{l}_{i}}$, at least as far as the real parts of these functions are concerned. In contrast, the imaginary part of $S$ can contribute more than the imaginary part of $F$ for very light squarks, i.e. $\sim 100 \mathrm{GeV}$.

Thus, if the squark sector has vanishing chirality-mixing terms, we expect the dominant contribution to $A_{S}^{\widetilde{l}_{i} \widetilde{l}_{i}}$ to be induced by the $t$-quark loop, followed by an in-generalsmaller contribution coming from the $\widetilde{t}$-squark loop, with exact size depending on $\hat{s}_{\text {min }}$ and the two $\widetilde{t}$-squark masses. Finally there is a much smaller contribution from the $\widetilde{b}$-squark loop, at least as far as $\widetilde{m}_{b_{i}} \simeq \widetilde{m}_{t_{i}}$ for $(i=1,2)$.

Since $\left(h_{E}\right)_{3,3}^{2} \cos \beta$ is also small, the production of same-chirality sleptons proceeds always through the D-term contributions to the couplings $g_{\mathcal{H} \widetilde{l}_{i} \widetilde{l}_{i}}$, for any of the values of $\tan \beta$ considered here $(\tan \beta \lesssim 3)$. Therefore, the size of $\left|\widetilde{A}_{S, \mathcal{H}} \widetilde{l}_{i} \widetilde{l}_{i}\right|$ from $\widetilde{t}$-squark exchange is expected to be in the same ballpark of the size of $\left|\widetilde{A}_{\mathrm{QC}}^{\widetilde{l}_{i} \widetilde{c}_{i}}\right|$ obtained before, except in the resonant region $\hat{s} \sim m_{\mathcal{H}}^{2}$, where $\left|\widetilde{A}_{S, \mathcal{H}}^{\tilde{l}_{i} \widetilde{l}_{i}}\right|$ has the chance to exceed $\left|\widetilde{A}_{\mathrm{QC}}^{\tilde{l}_{\mathrm{l}} \tilde{l}_{i}}\right|$ substantially. We have assumed here that also the slepton sector has vanishing chirality-mixing terms.

These observations are confirmed by the results shown in Figs. 6 and 7, where we plot the cross sections for the production of right-handed and left-handed sleptons, respectively, versus $\widetilde{m}_{q}$, which is defined in Sec. 2.2 . In both cases we give these results for the Higgs spectrum I and III. For spectrum I, we have used the values $m_{h}=98 \mathrm{GeV}, m_{H}=$ $162 \mathrm{GeV}$, and $\tan \alpha \sim-1$. In spectrum III, it is $m_{h}=114 \mathrm{GeV}$.

The Higgs-mediated cross sections, shown by the red and blue solid lines, are almost horizontal, at $\sim 0.2 \mathrm{fb}$ for the Higgs spectrum $\mathbf{I}$, and $\sim 0.1 \mathrm{fb}$ for the Higgs spectrum III. That is, in spite of the fact that spectrum III contributes with the exchange of $h$ only, whereas spectrum I contributes with $h$ and $H$, the value of the Higgs-exchange cross section is very similar in the two cases, provided the two scalar Higgs bosons in spectrum I are practically degenerate and have mass very similar to that of the light Higgs in spectrum III, i.e. $m_{h}^{\mathrm{I}} \sim m_{H}^{\mathrm{I}} \sim m_{h}^{\mathrm{III}}$. This is true for both, $\widetilde{A}_{\mathrm{H}}^{\widetilde{l}_{i}}$ and $\widetilde{A}_{\mathrm{H}}^{\widetilde{l}_{\mathrm{l}}} \widetilde{l}_{i}$. It is easy to convince oneself of this by inspecting the relevant couplings in Tables 2 and 3 . A splitting between $m_{h}^{\mathrm{I}}$ and $m_{H}^{\mathrm{I}}$, however, is in general present in spectrum $\mathbf{I}$, with size depending on the value of $\tan \beta$ and the squark spectrum. This accounts for the roughly $50 \%$ difference in the cross sections obtained for the Higgs spectrum I and III. Being practically independent 


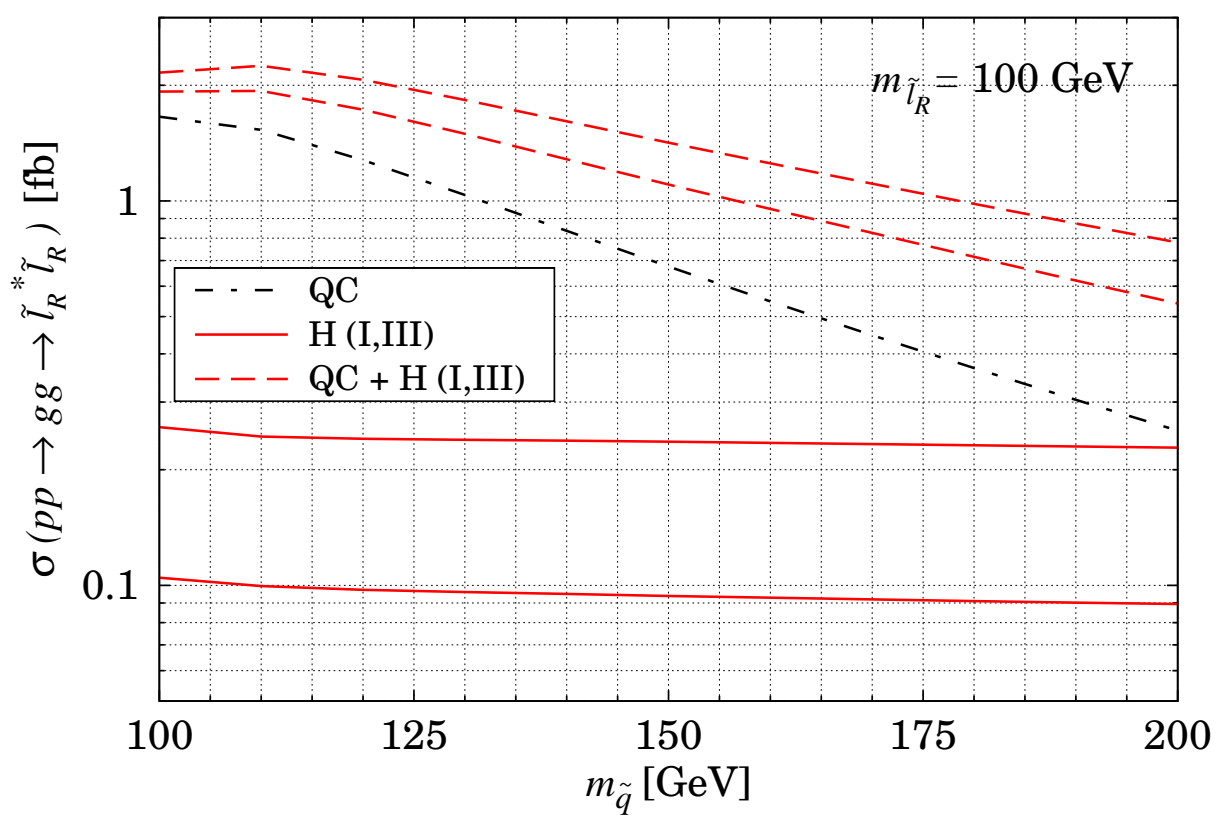

Figure 6: LHC cross section versus the parameter $\widetilde{m}_{q}$ in the squark spectrum A for the pair production of right-handed sleptons induced by: quartic couplings only (black dot-dashed line), Higgs exchange only (red solid lines, the upper one for the Higgs spectrum $\mathbf{I}$, the lower one for the spectrum III), and by both production mechanisms (red dashed lines).

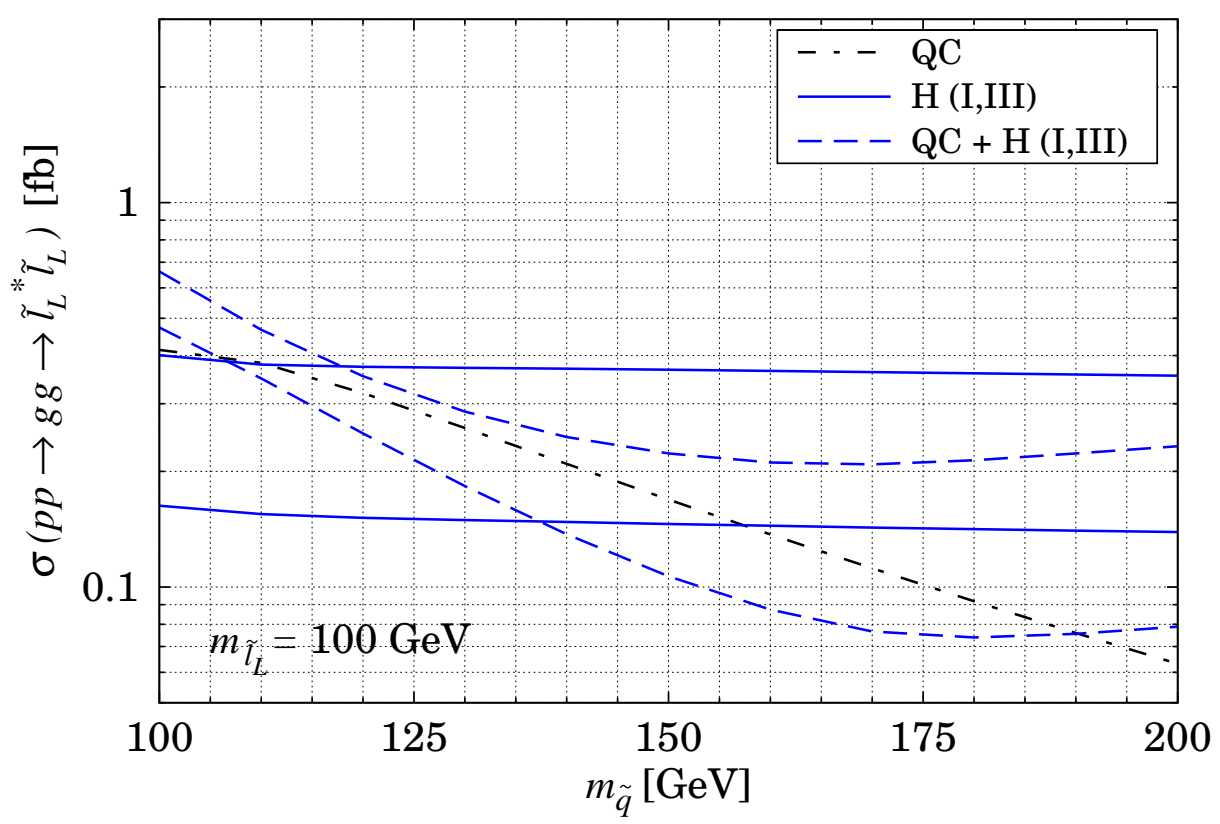

Figure 7: Same as in Fig. 6] for left-handed sleptons. 


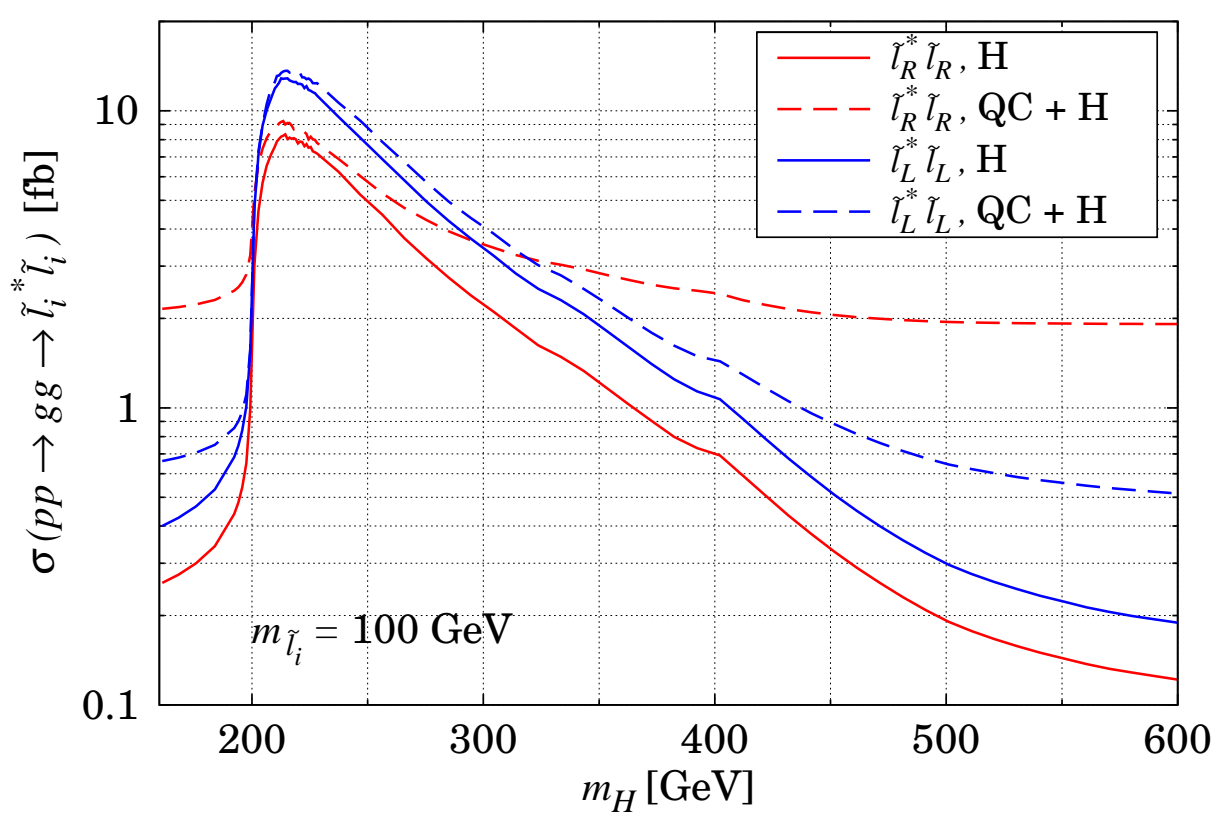

Figure 8: LHC cross sections for the production of right- and left-handed sleptons versus $m_{H}$. The solid lines show the cross sections from Higgs exchange only, the dashed lines from both, Higgs exchange and quartic couplings. For each of these two groups of curves, those which are lower at the peak of the resonance correspond to the production of right-handed sleptons, the higher curves, to that of left-handed sleptons. The squark spectrum A with $\widetilde{m}_{q}=100 \mathrm{GeV}$, which induces $\widetilde{m}_{t_{1}} \simeq 200 \mathrm{GeV}$ (see text), was used for the evaluation of all squark loops.

of $\widetilde{m}_{q}$, the Higgs-mediated cross sections show explicitly that the contribution from $\widetilde{A}_{\mathrm{H}}^{l_{i} \widetilde{l}_{i}}$ is small compared to that from $A_{\mathrm{H}}^{\tilde{l}_{i} \widetilde{l}_{i}}$, as already anticipated.

The quartic-coupling cross sections are shown by the black dot-dashed lines. They coincide with those in Fig. 2 .

The total gluon-initiated cross sections are given by the two red (blue) dashed lines, the upper one for spectrum I, the lower one for spectrum III. Although the interference effects can be significant, they are similar in size to the corresponding ones induced by quartic couplings only. We remind that the squark spectrum $\mathbf{A}$ used for these figures was chosen in such a way to maximize the size of $\left|\widetilde{A}_{\mathrm{QC}}^{\widetilde{l}_{\mathrm{l}}}\right| \widetilde{l}_{i}$, but not necessarily that of $\left|\widetilde{A}_{S, \mathcal{H}}^{\widetilde{l}_{i} \widetilde{l}_{i}}\right|$. Indeed, the value of the lightest $\widetilde{t}$-squark in the loop ranges between $200-260 \mathrm{GeV}$ when $\widetilde{m}_{q}$ spans the interval $100-200 \mathrm{GeV}$.

Enhancements of these cross sections are possible in the region in which the Higgs boson $H$ and/or $A$ are resonant. The cross sections in this region are very sensitive to the widths $\Gamma_{H}$, which we have evaluated with the program CPSUPERH [14].

We plot in Fig. 8 the cross sections obtained for the production of same-chirality sleptons at the lowest point in the squark spectrum $\mathbf{A}$, as a function of $m_{H}$. The smallest value of $m_{H}$ is that of the Higgs spectrum I of Figs. 6 and 7 , the largest value, is quite 
close to the $m_{H}$ of spectrum III in these same figures. No exchange of the pseudoscalar Higgs boson $A$ is possible in this case. The production of right-handed sleptons is shown by red lines, that of left-handed sleptons by blue lines. The solid lines show the cross sections induced by Higgs exchange only, the dashed lines show the cross sections due to both production mechanisms. The enhancement over the values of the cross sections shown in Figs. 6 and 7 is considerable for a rather large range $m_{H}$. Although the $\widetilde{t}$-squark loop gives a subdominant contribution to the Higgs-exchange cross section with respect to $t$-quark loop, a smeared peak is nevertheless visible at $m_{H} \sim 2 \widetilde{m}_{t_{1}}$. Far less visible is that at $m_{H} \sim 2 m_{t}$, because of the smoother behaviour at $\hat{s} \sim 2 m_{t}$ of the real and imaginary part of the function $F$, with respect to that in the case of the function $S$. (See Fig. [5.) At the peak of these resonances, the production cross sections for a pair of right-handed sleptons are roughly twice as large as those for a pair of left-handed sleptons. This is mainly due to the fact that $\left|d^{H_{i}^{0} H_{i}^{0} \widetilde{l}_{R} \widetilde{l}_{R}} / d^{H_{i}^{0} H_{i}^{0}, \widetilde{L}_{L} \widetilde{l}_{L}}\right|=\left(g_{2}^{2} / 4-g_{1}^{2} / 4\right) /\left(g_{1}^{2} / 2\right)$, for $(i=u, d)$.

As in the case of those shown in Fig. 2, also these cross sections apply to the production of slepton of first and second generation, as well as those of third generation for low $\tan \beta$. A value $\tan \beta=3$, for example, suppresses the $\tau$-lepton Yukawa couplings sufficiently to reduce $r_{\tau}$ (see discussion in Sec. (2.2) to be about few percent, as requested in the squark spectrum A. A moderate cancellation between $\left(A_{L}\right)_{3,3}$ and $\mu \tan \beta$ takes place in the chirality mixing terms of the $\widetilde{\tau}$ sector, for a value of $|\mu| \simeq 100 \mathrm{GeV}$ and $\left|\left(A_{L}\right)_{3,3}\right| \simeq$ $200 \mathrm{GeV}$, presumably not too large to jeopardize the metastability of the color- and chargepreserving vacuum.

The suppression of $\left(h_{E}\right)_{3,3}$, however, is not sufficient to reduce the couplings $g_{H \widetilde{\tau}_{R} \tilde{\tau}_{L}}$, $g_{A \widetilde{\tau}_{R} \tilde{\tau}_{L}}$ and their conjugates, in which the parameters $\left(A_{L}\right)_{3,3}$ and $\mu$ appear in different combinations, to be negligible with respect, for example, to the couplings $g_{H \widetilde{\tau}_{R} \widetilde{\tau}_{R}}, g_{A \widetilde{\tau}_{R} \widetilde{\tau}_{R}}$ coming mainly from D-terms.

Thus, the production of opposite chirality $\widetilde{\tau}$-sleptons is possible even for chiralitymixing terms in both, the $\widetilde{\tau}$-slepton mass matrix as well as in squark mass matrices that are nearly vanishing, and for a relatively low value of $\tan \beta$. This is in contrast to the quartic-coupling case, in which the the production of opposite-chirality $\widetilde{\tau}$ sleptons requires a large value of $\tan \beta$.

Indeed, not only is the production of opposite-chirality $\widetilde{\tau}$ 's possible in this case, but it has a cross section larger than that shown for the same spectrum in Fig. 8, at least in the resonant Higgs region. This is because both the two heavier Higgs bosons, $H$ and $A$, can mediate the $t$-quark contribution to the cross section. Notice that, because no chirality-mixing terms are present in the squark sector, the exchange of the pseudoscalar Higgs boson is possible only with the $t$-quark loop. Given the sharp behaviour of the function $P$ shown in Fig. 5 , the peak at the $t$-quark threshold is now considerably more pronounced than in the case of the cross section of Fig. 8 with exchange of only the Higgs boson $H$.

The values of the cross section that this last figure shows on top of the Higgs resonance are now quite interesting. We expect these values, as well as those for the production of same-chirality sleptons, to increase even further for nonvanishing chirality-mixing terms in 


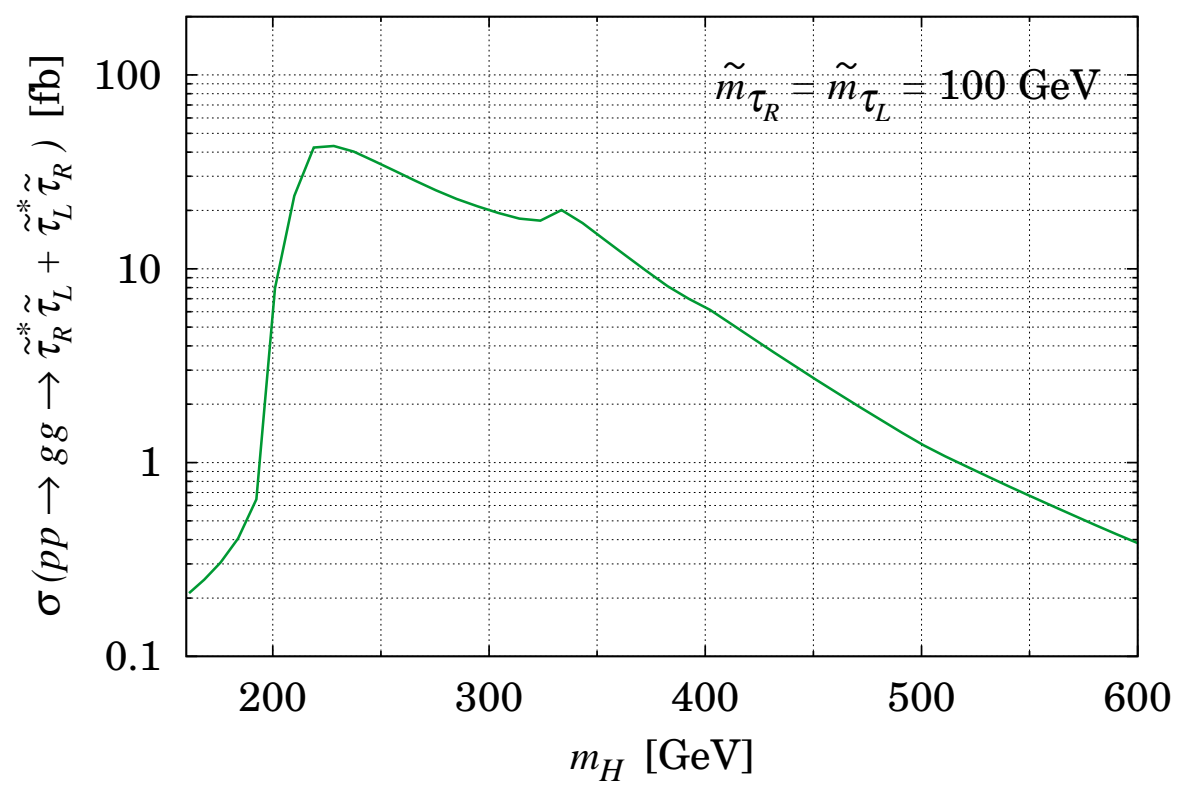

Figure 9: LHC cross section for the production of opposite-chirality $\widetilde{\tau}$ 's versus $m_{H}$, obtained at the lowest point of the squark spectrum $\mathbf{A}$ for $\tan \beta=3$. The contribution from quartic couplings is identically vanishing.

the squark sector. Depending on the values of the trilinear soft parameters, $\mu$, and $\tan \beta$, also the $\widetilde{b}$-squark contribution may become competitive with that due to the $\widetilde{t}$-squark and possibly also the $t$-quark.

The results shown here are rather promising. We believe these cross sections should be studied in full generality for more realistic sfermion spectra than the one considered here, and for all values of $\tan \beta$, in order to ascertain the possibility of probing, through them, important elements of the supersymmetric parameter space.

\section{Summary and outlook}

We have discussed the possibility of producing slepton pairs through gluon fusion. Our guiding motivation was that of assessing the impact that the quartic couplings $\widetilde{q}^{*} \widetilde{q} \widetilde{l}^{*} \widetilde{l}$ can have at the loop level for slepton production. If unmistakably detected, coupling such as these could provide an important confirmation of supersymmetric models.

We have found that the corresponding cross sections are small. That due to the only sizable quartic coupling from F-terms is small because it is proportional to the square of the product of the Yukawa couplings of the $b$ quark and the $\tau$ lepton. The slepton produced are in this case $\widetilde{\tau}$ sleptons. The values of these couplings can be increased for large $\tan \beta$. Nevertheless, the cross section gets in general penalized by the mixing effects in the slepton and the squark sector, in spite of the fact that this last mixing is necessary 
to produce the effective coupling $g g \widetilde{l^{*}} \widetilde{l}$.

The cross section for slepton production is small also when it is induced by quartic couplings from D-terms. The reason is due to a peculiar cancellation mechanism. Like the Glashow-Iliopoulos-Maiani (GIM) mechanism cancels the contributions from degenerate fermions of up or down-type quarks with different flavour in loop-induced flavourchanging-neutral-current processes, this mechanism cancels the contributions to slepton production from squarks that are degenerate within each generation. The two cancellation mechanisms have however quite different origins. The GIM mechanism is due to the unitarity of the Cabibbo-Kobayashi-Maskawa mixing matrix, whereas the cancellation observed in slepton production is due to the fact that the sum of the SM charges of all quark fields in each family vanishes identically.

In contrast, what we had hoped to be only a supersymmetric background to the gluon-fusion production of slepton pairs through quartic couplings, i.e. the gluon-fusion production with the exchange of a Higgs boson, turns out to give interesting results. The cross section can reach up to $\mathcal{O}(10) \mathrm{fb}$ if the exchanged Higgs boson becomes resonant. This value is obtained at the peak of the resonance, for a slepton mass of $100 \mathrm{GeV}$, for vanishing chirality-mixing terms in the squark sector. In this case, the dominant contribution comes from the $t$-quark loop that of the $\widetilde{t}$-squark is subdominant, and the $\widetilde{b}$-squark contribution is practically negligible. The same spectrum gives a cross section up to $40 \mathrm{fb}$ for the production of a pair of opposite-chirality $\widetilde{\tau}$ 's even for $\tan \beta=3$ and chirality mixing terms in the $\widetilde{\tau}$ sector practically negligible. Notice with the same type of slepton spectrum, only same-chirality sleptons can be produced through the Drell-Yan production mechanism [6].

We have not given explicit values of the cross sections when chirality-mixing terms in the squarks and slepton sectors are nonvanishing, and for large values of $\tan \beta$, but we have argued why we expect the cross section to increase in this case, at least for sleptons still relatively light. A full-fledged analysis should be performed with more realistic squark spectra than those used here, which were devised to maximize the gluonfusion cross sections from quartic-couplings. Moreover, when $\tan \beta$ is large, the tree-level process $b \bar{b} \rightarrow$ mediated by Higgs boson exchange, should also be added, incoherently, to the previous processes, in order to give a correct assessment of the values that the gluoninitiated cross section can reach. This is because the $b$ quark is contained in the proton via a gluon and the $b \bar{b}$-initiated cross section is of the same order in $\alpha_{s}$ than those studied here.

Finally, where the gluon-fusion mechanism of slepton production stands with respect to the Drell-Yan production depends also strongly on the role played by the QCD corrections to the two types of processes. Higher-order QCD corrections should indeed be included, together with SUSY-QCD corrections. The QCD corrections for the gluonfusion mechanism are as those for the production of Higgs bosons [15, 16]. They are known to be large, more than the corrections that QCD induces in the case of the DrellYan production [17]. The SUSY-QCD corrections are also well known [18]. At large tan $\beta$ those to the Higgs boson vertex may be well approximated [19] by the usual Yukawa 
coupling corrections.

It is widely believed that, if at all, sleptons will be discovered at the LHC through cascade decays. The production of a pair of sleptons, if kinematically allowed, will provide their direct mass measurement. Not much more than this can be learned from the DrellYan production. The gluon-fusion production mechanism, in this respect is potentially much more interesting since it can provide valuable information about $A, \mu$, and $\tan \beta$, and confirmations about the Higgs spectrum. Both mechanisms contribute to the pair production of sleptons, and the total cross section may be larger than previously thought, at least for resonant Higgs exchange. More detailed studies on this subject are clearly needed.

Going back to the issue of quartic couplings, an extended gauge structure, for example with an anomalous $\mathrm{U}(1)_{Y}$ in which the cancellation mechanism can be avoided, may help with their detection. It is however possible that the hadron collider environment is not the best suited for this purpose. The possibilities that a linear collider may offer in this respect should be explored.

In the case of its photon-collider option, for example, it is easy to find a situation that parallels very closely the one described here of a pair of sleptons, which, in spite of not being sensitive to strong interactions, can nevertheless couple at the one-loop level to a pair of gluons, thanks to their quartic couplings to squark pairs. The situation that immediately comes to mind is that of a pair of neutral scalars that can be coupled at the one-loop level to two photons thanks to their quartic couplings to a pair of charged scalars.

If the two neutral particles are supersymmetric Higgs bosons, the situation can actually be much better than that of slepton production discussed here. Indeed, the cancellation mechanism above described, which does work if the charged particles exchanged in the loop are squarks, is violated when these scalars are sleptons. This is simply due to the fact that the neutral components of the $\mathrm{SU}(2)_{L}$ slepton doublets cannot couple to photons.

The production of a pair of supersymmetric Higgs bosons in a photon collider was already considered in several papers [20, 21]. The charged slepton contribution to the loop, is mentioned only in Ref. [21]. It is, however, difficult to understand from this analysis the relevance of this contribution. It may remain buried under possibly larger contributions, not only those due to the exchange of Higgs bosons, but also those coming from box diagrams, that have no correspondent in our case of slepton production. A check of the relative size of all these contributions would probably be worthwhile.

\section{Acknowledgements}

The authors thank E. Accomando, B. Allanach, K. Fujii, J.S. Lee, K. Odagiri, N. Okamura, N. Polonsky, and K. Yokoya for stimulating discussions and inputs, D.W. Jung for initial collaboration, and M. Spira for a critical reading of the manuscript. F. B. acknowledges the NCTS-KEK Exchange program, which has made this collaboration possible and the hospitality extended to her by the Yukawa Institute in Kyoto, during the YKIS 2009. 
The work of F.B. was partially supported by the Excellency Research Project of National Taiwan University, Taiwan: "Mass generation, heavy flavours, neutrinos at the particle physics frontier", grant No 97R0066-60. K.H. is supported in part by the Grant-in-Aid for scientific research (No. 20340064) from MEXT, Japan.

\section{References}

[1] H. Murayama, Confusing signals of Supersymmetry, International Linear Collider Workshop (LCWS2000), Oct. 24-28, 2000, Fermilab, http://conferences.fnal.gov/lcws2000/web/P3_Murayama/index.html.

[2] L. T. Wang and I. Yavin, A Review of Spin Determination at the LHC, Int. J. Mod. Phys. A 23 (2008) 4647

[3] Papers cited in Ref [2] and in:

F. Boudjema and R. K. Singh, A model independent spin analysis of fundamental particles using azimuthal asymmetries, JHEP 0907 (2009) 028,

S. Y. Choi, K. Hagiwara, H. U. Martyn, K. Mawatari and P. M. Zerwas, Spin analysis of supersymmetric particles, Eur. Phys. J. C 51 (2007) 753

[4] F. del Aguila and L. Ametller, On the detectability of sleptons at large hadron colliders, Phys. Lett. B 261 (1991) 326

[5] M. Bisset, S. Raychaudhuri and P. Roy, Higgs-mediated Slepton Pair-production at the Large Hadron Collider, arXiv:hep-ph/9602430

[6] E. Eichten, I. Hinchliffe, K. D. Lane and C. Quigg, Super Collider Physics, Rev. Mod. Phys. 56 (1984) 579 [Addendum-ibid. 58 (1986) 1065],

S. Dawson, E. Eichten and C. Quigg, Search For Supersymmetric Particles In HadronHadron Collisions, Phys. Rev. D 31 (1985) 1581,

P. Chiappetta, J. Soffer and P. Taxil, Spin Asymmetries For Scalar Leptons From W And $Z$ Decay In P Anti-P Collisions, Phys. Lett. B 162 (1985) 192,

H. Baer, C. h. Chen, F. Paige and X. Tata, Detecting sleptons at hadron colliders and supercolliders, Phys. Rev. D 49 (1994) 3283

[7] M. Claudson, L. J. Hall and I. Hinchliffe, Low-Energy Supergravity: False Vacua And Vacuous Predictions, Nucl. Phys. B 228 (1983) 501,

J. A. Casas, A. Lleyda and C. Munoz, Strong constraints on the parameter space of the MSSM from charge and color breaking minima, Nucl. Phys. B 471 (1996) 3

[8] A. Kusenko, P. Langacker and G. Segre, Phase Transitions and Vacuum Tunneling Into Charge and Color Breaking Minima in the MSSM, Phys. Rev. D 54 (1996) 5824,

U. Sarid, Tools for tunneling, Phys. Rev. D 58 (1998) 085017 arXiv:hep-ph/9804308],

F. Borzumati, G. R. Farrar, N. Polonsky and S. D. Thomas, Soft Yukawa couplings in supersymmetric theories, Nucl. Phys. B 555 (1999) 53

[9] K. Hikasa, Supersymmetric Standard Model for Collider Physicists, to be requested to the author,

S. P. Martin, A Supersymmetry Primer, arXiv:hep-ph/9709356 
[10] J. Pumplin, D. R. Stump, J. Huston, H. L. Lai, P. Nadolsky and W. K. Tung, New generation of parton distributions with uncertainties from global QCD analysis, JHEP 0207 (2002) 012

[11] S. Kawabata, A New Monte Carlo Event Generator For High-Energy Physics, Comput. Phys. Commun. 41 (1986) 127 and A New version of the multidimensional integration and event generation package BASES/SPRING, Comput. Phys. Commun. 88 (1995) 309

[12] M. Spira, QCD effects in Higgs physics, Fortsch. Phys. 46 (1998) 203

[13] A. Djouadi, The anatomy of electro-weak symmetry breaking. II: The Higgs bosons in the minimal supersymmetric model, Phys. Rept. 459 (2008) 1,

M. S. Carena et al. [Higgs Working Group Collaboration], Report of the Tevatron Higgs working group, arXiv:hep-ph/0010338

[14] J. S. Lee, A. Pilaftsis, M. S. Carena, S. Y. Choi, M. Drees, J. R. Ellis and C. E. M. Wagner, CPsuperH: A computational tool for Higgs phenomenology in the minimal supersymmetric standard model with explicit CP violation, Comput. Phys. Commun. 156 (2004) 283

[15] D. Graudenz, M. Spira and P. M. Zerwas, QCD corrections to Higgs boson production at proton proton colliders, Phys. Rev. Lett. 70 (1993) 1372,

M. Spira, A. Djouadi, D. Graudenz and P. M. Zerwas, Higgs boson production at the LHC, Nucl. Phys. B 453 (1995) 17,

R. V. Harlander and K. J. Ozeren, Top mass effects in Higgs production at next-to-next-toleading order QCD: virtual corrections, Phys. Lett. B 679 (2009) 467,

A. Pak, M. Rogal and M. Steinhauser, Virtual three-loop corrections to Higgs boson production in gluon fusion for finite top quark mass, Phys. Lett. B 679 (2009) 473,

S. Catani, D. de Florian, M. Grazzini and P. Nason, Soft-gluon resummation for Higgs boson production at hadron colliders, JHEP 0307 (2003) 028

[16] S. Dawson, A. Djouadi and M. Spira, QCD corrections to SUSY Higgs production: The Role of squark loops, Phys. Rev. Lett. 77 (1996) 16,

M. Muhlleitner and M. Spira, Higgs boson production via gluon fusion: Squark loops at NLO QCD, Nucl. Phys. B 790 (2008) 1,

R. Bonciani, G. Degrassi and A. Vicini, Scalar Particle Contribution to Higgs Production via Gluon Fusion at NLO, JHEP 0711 (2007) 095,

M. Muhlleitner, H. Rzehak and M. Spira, MSSM Higgs Boson Production via Gluon Fusion: The Large Gluino Mass Limit, JHEP 0904 (2009) 023

[17] W. Beenakker, M. Klasen, M. Kramer, T. Plehn, M. Spira and P. M. Zerwas, The Production of charginos / neutralinos and sleptons at hadron colliders, Phys. Rev. Lett. 83 (1999) 3780 [Erratum-ibid. 100 (2008) 029901],

M. Grazzini, The Drell-Yan process in NNLO QCD, arXiv:0908.1336 [hep-ph]

[18] R. V. Harlander and M. Steinhauser, Supersymmetric Higgs production in gluon fusion at next-to-leading order, JHEP 0409 (2004) 066,

R. V. Harlander and F. Hofmann, Pseudo-scalar Higgs production at next-to-leading order $S U S Y$-QCD, JHEP 0603 (2006) 050,

G. Degrassi and P. Slavich, On the radiative corrections to the neutral Higgs boson masses in the NMSSM, Nucl. Phys. B 825 (2010) 119, 
C. Anastasiou, S. Beerli and A. Daleo, The two-loop QCD amplitude $g g \rightarrow h, H$ in the Minimal Supersymmetric Standard Model, Phys. Rev. Lett. 100 (2008) 241806

[19] M. S. Carena, D. Garcia, U. Nierste and C. E. M. Wagner, Effective Lagrangian for the $\bar{t} b H^{+}$interaction in the MSSM and charged Higgs phenomenology, Nucl. Phys. B 577 (2000) 88 ,

J. Guasch, P. Hafliger and M. Spira, MSSM Higgs decays to bottom quark pairs revisited, Phys. Rev. D 68 (2003) 115001,

F. Borzumati, C. Greub and Y. Yamada, Beyond leading-order corrections to $\bar{B} \rightarrow X_{s} \gamma$ at large tan(beta): The charged-Higgs contribution, Phys. Rev. D 69 (2004) 055005, and Towards an exact evaluation of the supersymmetric $O($ alpha(s) tan(beta)) corrections to $\bar{B} \rightarrow X_{s} \gamma$, arXiv:hep-ph/0305063

D. Noth and M. Spira, Higgs Boson Couplings to Bottom Quarks: Two-Loop Supersymmetry-QCD Corrections, Phys. Rev. Lett. 101 (2008) 181801

[20] S. H. Zhu, Pseudoscalar Higgs boson pair production in photon photon collisions, J. Phys. G 24 (1998) 1703,

S. H. Zhu, C. S. Li and C. S. Gao, Lightest neutral Higgs pair production in photon photon collisions in the minimal supersymmetric standard model, Phys. Rev. D 58 (1998) 015006, G. J. Gounaris and P. I. Porfyriadis, The gamma gamma $\rightarrow$ AO AO process at a gamma gamma collider, Eur. Phys. J. C 18 (2000) 181

[21] Y. J. Zhou, W. G. Ma, H. S. Hou, R. Y. Zhang, P. J. Zhou and Y. B. Sun, Neutral Higgs boson pair production via gamma gamma collision in the minimal supersymmetric standard model at linear colliders, Phys. Rev. D 68 (2003) 093004 